NISSUNA UMANA INVESTIGAZIONE SI PUO DIMANDARE VERA SCIENZIA S'ESSA NON PASSA PER LE MATEMATICHE DIMOSTRAZIONI LEONARDO DA VINCI

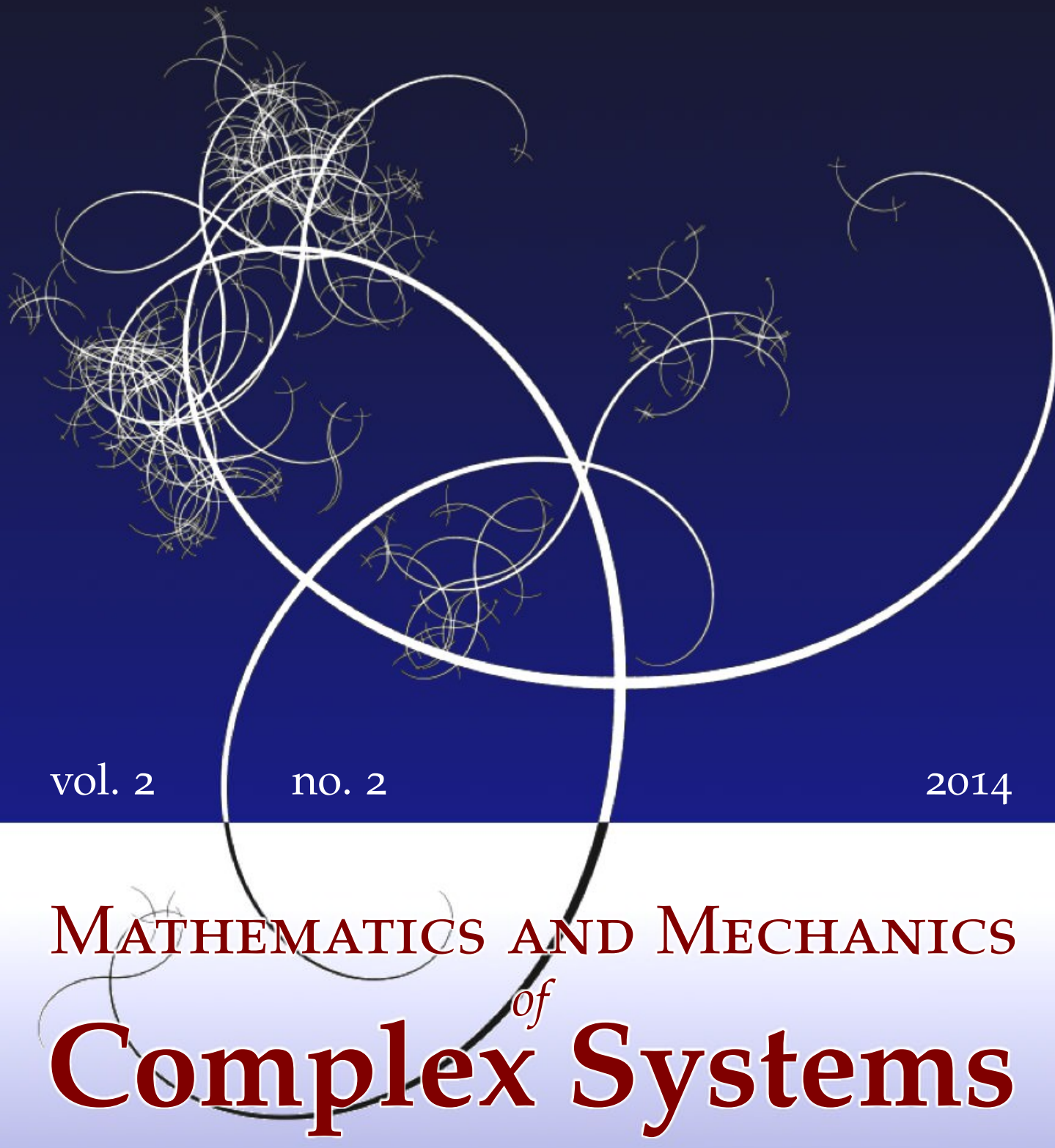

YAHYA BERREHILI AND JEAN-JACQUES MARIGO

THE HOMOGENIZED BEHAVIOR OF UNIDIRECTIONAL FIBER-REINFORCED COMPOSITE MATERIALS IN THE CASE OF DEBONDED FIBERS 


\title{
THE HOMOGENIZED BEHAVIOR OF UNIDIRECTIONAL FIBER-REINFORCED COMPOSITE MATERIALS IN THE CASE OF DEBONDED FIBERS
}

\author{
YAHYA BERREHILI AND JEAN-JACQUES MARIGO
}

\begin{abstract}
This paper is devoted to the analysis of the homogenized behavior of unidirectional composite materials once the fibers are debonded from (but still in contact with) the matrix. This homogenized behavior is built by an asymptotic method in the framework of the homogenization theory. The main result is that the homogenized behavior of the debonded composite is that of a generalized continuous medium with an enriched kinematics. Indeed, besides the usual macroscopic displacement field, the macroscopic kinematics contains two other scalar fields. The former one corresponds to the displacement of the matrix whereas the two latter ones correspond to the sliding and the rotation of the debonded fibers with respect to the matrix. Accordingly, new homogenized coefficients and new coupled equilibrium equations appear. This problem is addressed in a linear elastic three-dimensional setting.
\end{abstract}

\section{Introduction}

The use of unidirectional fiber-reinforced composite materials does not cease to grow in various domains and particularly in the domains of aerospace and aeronautics. This is due to their various properties and especially to their interesting mechanical behavior in terms of their specific effective stiffness in the direction of the fibers. (Throughout the paper, the word effective is a synonym of homogenized or macroscopic.) The effective elastic behavior of such composites is now well known and well modeled by the homogenization theory as long as the fibers are assumed to be perfectly bonded to the matrix [Léné 1984; Michel et al. 1999; Sánchez-Palencia 1980; Suquet 1982].

However, since their mechanical performance is considered optimal when the components remain bonded, it remains to evaluate the loss of performance when

\section{Communicated by Pierre Seppecher.}

This work was partially supported by the French Agence Nationale de la Recherche (ANR), under grant epsilon (BLAN08-2_312370) "Domain decomposition and multi-scale computations of singularities in mechanical structures".

MSC2000: 35C20, 35J20, 74B05, 74G10, 74Q15.

Keywords: homogenization, composite materials, debonding. 
the fibers are debonded. Of course, if one considers that the elastic behavior is due to the matrix alone, the specific stiffness drops drastically. But this type of estimate simply gives a lower bound to the stiffness and one must define more precisely the effective behavior of completely or partially debonded unidirectional composites.

Many works have been devoted to this task; see for instance [Bouchelaghem et al. 2007; Caporale et al. 2006; Gonzàlez and LLorca 2007; Greco 2009; Jendli et al. 2009; Kulkarni et al. 2009; Kushch et al. 2011; Léné and Leguillon 1982; Marigo et al. 1987; Matouš and Geubelle 2006; Moraleda et al. 2009; Teng 2010]. In general, these studies consist in replacing the perfect bond of the interface by some "cohesive law" or simply in removing the fibers when the debonding is complete. In any case, the calculation of the new homogenized mechanical coefficients is performed by considering the usual elementary problems set on the unit cell without reconsidering the general procedure of homogenization. However, when following the two-scale asymptotic approach, it appears that the argument used to obtain that the zero-order displacement field does not depend on the microscopic variable is no longer valid. Therefore, in the zone where the fibers are debonded, the macroscopic displacement field must be replaced by another "macroscopic" displacement field, corresponding to the independent displacement of the fibers [Berrehili and Marigo 2010]. Consequently, one must also construct the macroscopic problem which gives this additional field. That is the purpose of this paper.

Specifically, the paper is organized as follows. The next section is devoted to the setting of the problem: one considers a composite structure $\Omega$, constituted by a periodic distribution of elastic unidirectional fibers whose direction is $\boldsymbol{e}_{3}$ and embedded in an elastic matrix. In a part $\Omega_{c}$ of $\Omega$ the fibers are assumed to be bonded to the matrix whereas in the complementary part $\Omega_{d}$ they are assumed to be debonded but still in contact without friction with the matrix. We then formulate the elastostatic problem which contains the small parameter $\epsilon$ related to the size of the microstructure and which governs the displacement field $\boldsymbol{u}^{\epsilon}$ and the stress field $\boldsymbol{\sigma}^{\epsilon}$. The third section is devoted to the asymptotic analysis, i.e., the behavior of $\boldsymbol{u}^{\epsilon}$ and $\boldsymbol{\sigma}^{\epsilon}$ when $\epsilon$ goes to 0 . Following a two-scale approach, we first postulate that $\boldsymbol{u}^{\epsilon}$ and $\boldsymbol{\sigma}^{\epsilon}$ can be expanded in powers of $\epsilon$, the coefficients $\boldsymbol{u}^{i}(\boldsymbol{x}, \boldsymbol{y})$ and $\boldsymbol{\sigma}^{i}(\boldsymbol{x}, \boldsymbol{y})$ of the expansion being periodic functions of the microscopic coordinates $y$. We then obtain a sequence of variational equations in terms of the $\boldsymbol{u}^{i}$ and the $\boldsymbol{\sigma}^{i}$. These equations are sequentially solved to finally obtain the effective behavior of the composite in its bonded and debonded parts. In the fourth section, we study the properties of the effective model and, in particular, the properties of the effective coefficients provided by the solutions of linear elastic problems posed either on the bonded or on the debonded cell. Then, some examples are treated. We finally conclude giving some perspectives.

The summation convention on repeated indices is used throughout the paper. 
The set of real numbers, the set of $n$-dimensional vectors and the set of symmetric second-order $n$-dimensional tensors are, respectively, denoted by $\mathbb{R}, \mathbb{R}^{n}$ and $\mathbb{M}_{s}^{n}$. Vectors and second-order tensors are indicated by boldface letters, like $\boldsymbol{u}$ and $\boldsymbol{\sigma}$ for the displacement field and the stress field. Their components are denoted by italic letters, like $u_{i}$ and $\sigma_{i j}$. Fourth-order tensors as well as their components are indicated by sans-serif letters, like A or $\mathrm{A}_{i j k l}$ for the stiffness tensor. Such tensors are considered as linear maps acting on second-order tensors. The application of A to $\boldsymbol{\varepsilon}$ is denoted $A \boldsymbol{\varepsilon}$, with components $\mathrm{A}_{i j k l} \varepsilon_{k l}$. The inner product between two vectors or two tensors of the same order is indicated by a dot, like $\boldsymbol{a} \cdot \boldsymbol{b}$ which stands for $a_{i} b_{i}$ or $\boldsymbol{\sigma} \cdot \boldsymbol{\varepsilon}$ for $\sigma_{i j} \varepsilon_{i j}$. The symbol $\otimes$ denotes the tensor product and $\otimes_{s}$ denotes its symmetric part; i.e., $2 \boldsymbol{e}_{1} \otimes_{s} \boldsymbol{e}_{2}=\boldsymbol{e}_{1} \otimes \boldsymbol{e}_{2}+\boldsymbol{e}_{2} \otimes \boldsymbol{e}_{1}$.

In our frequent use of multiple scaling techniques, we adopt the related notation. For instance, $\boldsymbol{x}=\left(x_{1}, x_{2}, x_{3}\right)$ always denotes a macroscopic coordinate while $\boldsymbol{y}=\left(y_{1}, y_{2}\right)$ represents a microscopic one. Since the fibers are oriented along the direction $\boldsymbol{e}_{3}$, we distinguish the longitudinal coordinate $x_{3}$ from the transversal coordinates $\boldsymbol{x}^{\prime}=\left(x_{1}, x_{2}\right)$. Latin indices run from 1 to 3 , while Greek indices run from 1 to 2 . When a spatial (scalar, vectorial or tensorial) field depends both on $\boldsymbol{x}$ and $\boldsymbol{y}$, the partial derivative with respect to one of the coordinates appears explicitly as an index: for example, $\operatorname{div}_{x} \sigma$ and $\varepsilon_{x}(\boldsymbol{v})$ denote, respectively, the divergence of the stress tensor field $\sigma$ and the symmetric gradient of the vector field $v$ with respect to $\boldsymbol{x}$, while $\operatorname{div}_{\boldsymbol{y}} \sigma$ and $\varepsilon_{\boldsymbol{y}}(\boldsymbol{v})$ are the corresponding derivatives with respect to $\boldsymbol{y}$ :

$$
\begin{gathered}
\operatorname{div}_{\boldsymbol{x}} \boldsymbol{\sigma}(\boldsymbol{x}, \boldsymbol{y})=\frac{\partial \sigma_{i j}}{\partial x_{j}}(\boldsymbol{x}, \boldsymbol{y}) \boldsymbol{e}_{i}, \quad \operatorname{div}_{\boldsymbol{y}} \boldsymbol{\sigma}(\boldsymbol{x}, \boldsymbol{y})=\frac{\partial \sigma_{i \beta}}{\partial y_{\beta}}(\boldsymbol{x}, \boldsymbol{y}) \boldsymbol{e}_{i}, \\
\boldsymbol{\varepsilon}_{\boldsymbol{x}}(\boldsymbol{v})(\boldsymbol{x}, \boldsymbol{y})=\left(\frac{\partial v_{j}}{\partial x_{i}}(\boldsymbol{x}, \boldsymbol{y})+\frac{\partial v_{i}}{\partial x_{j}}(\boldsymbol{x}, \boldsymbol{y})\right) \boldsymbol{e}_{i} \otimes_{s} \boldsymbol{e}_{j}, \\
\boldsymbol{\varepsilon}_{\boldsymbol{y}}(\boldsymbol{v})(\boldsymbol{x}, \boldsymbol{y})=\left(\frac{\partial v_{\alpha}}{\partial y_{\beta}}(\boldsymbol{x}, \boldsymbol{y})+\frac{\partial v_{\beta}}{\partial y_{\alpha}}(\boldsymbol{x}, \boldsymbol{y})\right) \boldsymbol{e}_{\alpha} \otimes_{s} \boldsymbol{e}_{\beta}+\frac{\partial v_{3}}{\partial y_{\alpha}}(\boldsymbol{x}, \boldsymbol{y}) \boldsymbol{e}_{\alpha} \otimes_{s} \boldsymbol{e}_{3} .
\end{gathered}
$$

On a surface $\boldsymbol{I}$ across which a field $f$ is discontinuous, we denote by $\llbracket f \rrbracket$ its jump discontinuity.

\section{Statement of the problem}

We consider a heterogeneous elastic body whose natural reference configuration is a bounded open domain $\Omega$ of $\mathbb{R}^{3}$ with a smooth boundary $\partial \Omega$. We denote by $\left(\boldsymbol{e}_{1}, \boldsymbol{e}_{2}, \boldsymbol{e}_{3}\right)$ the canonical basis of $\mathbb{R}^{3}$ and by $\left(x_{1}, x_{2}, x_{3}\right)$ the coordinates of a point $\boldsymbol{x} \in \Omega$. The body is made of two isotropic linearly elastic materials, called the fibers and the matrix, whose Lamé coefficients and mass density are, respectively, $\left(\lambda_{f}, \mu_{f}, \rho_{f}\right)$ and $\left(\lambda_{m}, \mu_{m}, \rho_{m}\right)$. The fibers are aligned in the direction $\boldsymbol{e}_{3}$ and have a circular cross-section with radius $\epsilon R$. They are periodically distributed in the 


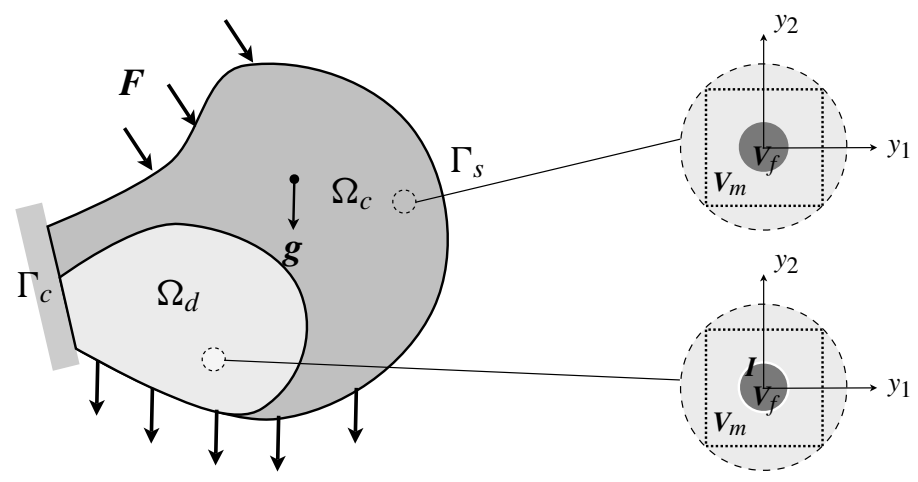

Figure 1. The composite structure and the two periodic cells.

matrix, $\epsilon \boldsymbol{a}$ and $\epsilon \boldsymbol{b}$ being the two vectors of the plane $\left(\boldsymbol{e}_{1}, \boldsymbol{e}_{2}\right)$ characterizing the periodicity. The number of fibers is large so that the dimensionless parameter $\epsilon$ characterizing the fineness of the microstructure (for instance, the ratio between the spatial period and the size of the structure) is small. The domain occupied by the fibers is $\Omega_{f}^{\epsilon}$, that occupied by the matrix is $\Omega_{m}^{\epsilon}$, while the set of all interfaces between fibers and matrix is $\boldsymbol{I}^{\epsilon}$. Accordingly, one has

$$
\Omega=\Omega_{f}^{\epsilon} \cup \boldsymbol{I}^{\epsilon} \cup \Omega_{m}^{\epsilon} .
$$

The fibers are perfectly bonded in a part $\Omega_{c}$ of $\Omega$ and debonded in the complementary part $\Omega_{d}$; see Figure 1 . Both parts contain a large number of fibers and will be considered as given and independent of $\epsilon$. Moreover we assume that in $\Omega_{d}$ the fibers remain in contact with the matrix but can slip without friction. Accordingly, denoting by

$$
\boldsymbol{I}_{c}^{\epsilon}=\Omega_{c} \cap \boldsymbol{I}^{\epsilon}, \quad \boldsymbol{I}_{d}^{\epsilon}=\Omega_{d} \cap \boldsymbol{I}^{\epsilon},
$$

respectively, the bonded and debonded interfaces, the interface conditions in terms of the displacement and the stress fields read as

$$
\left\{\begin{array}{cc}
\llbracket u \rrbracket=0, \quad \llbracket \sigma \rrbracket n=0 & \text { on } \boldsymbol{I}_{c}^{\epsilon}, \\
\llbracket u \rrbracket \cdot n=0, \quad \llbracket \sigma \rrbracket n \cdot n=0, \quad \boldsymbol{\sigma} n \wedge \boldsymbol{n}=0 & \text { on } \boldsymbol{I}_{d}^{\epsilon} .
\end{array}\right.
$$

In (6), $\boldsymbol{n}$ is the outer normal to the fiber at an interface and the brackets denote the jump of the involved field across the interface. The conditions on $\boldsymbol{I}_{c}^{\epsilon}$ mean that the displacement and the vector stress are continuous; the conditions on $\boldsymbol{I}_{d}^{\epsilon}$ mean that the normal displacement and the normal stress are continuous while the shear stress vanishes.

Remark 1. In the above conditions on the interface between the fibers and the matrix after debonding, we assume that contact always occurs without friction. This allows us to treat linear elastic problems and then the analysis is simplified. 
It would be easy to follow the same procedure by assuming that the fibers are no longer in contact with the matrix after debonding. It is more difficult to consider unilateral frictionless contact conditions where the contact conditions depend on the sign of the normal stress. That leads to nonlinear (but still elastic) problems where the superposition principle can no longer be used. Much more difficult is the case where the contact occurs with friction. Then the effective behavior is no longer elastic and one must introduce internal variables. All these more elaborated cases are outside the scope of this didactic paper and will be the subject of future works.

The body is submitted to a specific body force density $\boldsymbol{g}$ (independent of $\epsilon$ ). The part $\Gamma_{c}$ of the boundary $\partial \Omega$ is fixed while the complementary part $\Gamma_{s}=\partial \Omega \backslash \Gamma_{c}$ is submitted to a surface force density $\boldsymbol{F}$ (independent of $\epsilon$ ).

We are now in a position to set the problem which governs the response of the body at equilibrium under the given loading. For a fixed $\epsilon>0$, the problem consists in finding a displacement field $u^{\epsilon}$ and a stress field $\sigma^{\epsilon}$, such that:

Equilibrium: $\begin{cases}\operatorname{div} \sigma^{\epsilon}+\rho_{f} g=0 & \text { in } \Omega_{f}^{\epsilon}, \\ \operatorname{div} \sigma^{\epsilon}+\rho_{m} g=0 & \text { in } \Omega_{m}^{\epsilon},\end{cases}$

Constitutive relations: $\quad \begin{cases}\sigma^{\epsilon}=\lambda_{f} \operatorname{div} \boldsymbol{u}^{\epsilon} \delta+2 \mu_{f} \boldsymbol{\varepsilon}\left(\boldsymbol{u}^{\epsilon}\right) & \text { in } \Omega_{f}^{\epsilon}, \\ \sigma^{\epsilon}=\lambda_{m} \operatorname{div} \boldsymbol{u}^{\epsilon} \boldsymbol{\delta}+2 \mu_{m} \boldsymbol{\varepsilon}\left(\boldsymbol{u}^{\epsilon}\right) & \text { in } \Omega_{m}^{\epsilon},\end{cases}$

Compatibility: $\quad 2 \boldsymbol{\varepsilon}\left(\boldsymbol{u}^{\epsilon}\right)=\nabla \boldsymbol{u}^{\epsilon}+\nabla^{T} \boldsymbol{u}^{\epsilon} \quad$ in $\Omega_{f}^{\epsilon} \cup \Omega_{m}^{\epsilon}$,

Boundary conditions: $\left\{\begin{aligned} \boldsymbol{u}^{\epsilon} & =\mathbf{0} & & \text { on } \Gamma_{c}, \\ \boldsymbol{\sigma}^{\epsilon} \boldsymbol{n} & =\boldsymbol{F} & & \text { on } \Gamma_{s},\end{aligned}\right.$

Interface conditions: $\left\{\begin{array}{cc}\llbracket \boldsymbol{u}^{\epsilon} \rrbracket=\mathbf{0}, \quad \llbracket \boldsymbol{\sigma}^{\epsilon} \rrbracket \boldsymbol{n}=\mathbf{0} & \text { on } \boldsymbol{I}_{c}^{\epsilon}, \\ \llbracket u_{n}^{\epsilon} \rrbracket=0, \quad \boldsymbol{\sigma}^{\epsilon} \boldsymbol{n}=\sigma_{n n}^{\epsilon} \boldsymbol{n}, \quad \llbracket \sigma_{n n}^{\epsilon} \rrbracket=0 & \text { on } \boldsymbol{I}_{d}^{\epsilon} .\end{array}\right.$

In (8), $\delta$ is the identity tensor with $\delta_{i j}=1$ when $i=j$ and $\delta_{i j}=0$ when $i \neq j$. This set of equations constitutes a linear boundary value problem which can be written in a variational form as follows.

Let $\mathscr{C}^{\epsilon}$ be the linear space of kinematically admissible displacement fields; i.e.,

$$
\mathscr{C}^{\epsilon}=\left\{\boldsymbol{v} \in H^{1}\left(\Omega \backslash \boldsymbol{I}_{d}^{\epsilon} ; \mathbb{R}^{3}\right): \llbracket \boldsymbol{v} \rrbracket \cdot \boldsymbol{n}=\mathbf{0} \text { on } \boldsymbol{I}_{d}^{\epsilon}, \boldsymbol{v}=\mathbf{0} \text { on } \Gamma_{c}\right\},
$$

let $\mathrm{f}^{\epsilon}$ be the continuous linear form associated with the applied forces; i.e.,

$$
f^{\epsilon}(v)=\int_{\Omega_{f}^{\epsilon}} \rho_{f} \boldsymbol{g} \cdot \boldsymbol{v} d x+\int_{\Omega_{m}^{\epsilon}} \rho_{m} \boldsymbol{g} \cdot \boldsymbol{v} d x+\int_{\Gamma_{s}} \boldsymbol{F} \cdot \boldsymbol{v} d \Gamma \quad \text { for } \boldsymbol{v} \in \mathscr{C}^{\epsilon},
$$

and let $a^{\epsilon}$ be the bilinear continuous form associated with the elastic energy; i.e.,

$$
\mathrm{a}^{\epsilon}(\boldsymbol{u}, \boldsymbol{v})=\int_{\Omega_{f}^{\epsilon}} \mathrm{A}^{f} \boldsymbol{\varepsilon}(\boldsymbol{u}) \cdot \boldsymbol{\varepsilon}(\boldsymbol{v}) d x+\int_{\Omega_{m}^{\epsilon}} \mathrm{A}^{m} \boldsymbol{\varepsilon}(\boldsymbol{u}) \cdot \boldsymbol{\varepsilon}(\boldsymbol{v}) d x .
$$


In (14), $\mathrm{A}^{f}$ and $\mathrm{A}^{m}$ stand for the fourth-order elasticity tensors of the fibers and the matrix, respectively; i.e.,

$$
\mathrm{A}_{i j k l}^{f, m}=\lambda_{f, m} \delta_{i j} \delta_{k l}+\mu_{f, m}\left(\delta_{i k} \delta_{j l}+\delta_{i l} \delta_{j k}\right) .
$$

Then $\boldsymbol{u}^{\epsilon}$ must satisfy the variational problem

$$
\text { find } \boldsymbol{u}^{\epsilon} \in \mathscr{C}^{\epsilon} \quad \text { such that } a^{\epsilon}\left(\boldsymbol{u}^{\epsilon}, \boldsymbol{v}\right)=\mathrm{f}^{\epsilon}(\boldsymbol{v}) \text { for all } \boldsymbol{v} \in \mathscr{C}^{\epsilon},
$$

and $\sigma^{\epsilon}$ is the associated stress field given in terms of the strain field by (8). The existence and the uniqueness of the solution $\boldsymbol{u}^{\epsilon}$ of (16) is guaranteed provided that the boundary $\Gamma_{c}$ is such that there does not exist any (nonzero) rigid displacement which is kinematically admissible. Specifically, let us denote by $\mathscr{R}^{\epsilon}$ the set of displacement fields which are both kinematically admissible and corresponding to a null strain field; i.e.,

$$
\mathscr{R}^{\epsilon}=\left\{\boldsymbol{v} \in \mathscr{C}^{\epsilon}: \boldsymbol{\varepsilon}(\boldsymbol{v})=\mathbf{0} \text { in } \Omega \backslash \boldsymbol{I}_{d}^{\epsilon}\right\} .
$$

By standard arguments, we have:

Proposition 1. Under the condition that $\mathscr{R}^{\epsilon}=\{\mathbf{0}\}$ and that the density of forces $\boldsymbol{g}$ and $\boldsymbol{F}$ are smooth enough, the variational problem (16) admits a unique solution $\boldsymbol{u}^{\epsilon}$.

The necessary and sufficient condition above for the existence and the uniqueness of the solution depends in general both on $\Gamma_{c}$ and $\Omega_{d}$. However, the existence of a solution is guaranteed if $\mathscr{R}^{\epsilon}=\{\mathbf{0}\}$, that is, if no rigid displacements are allowed. We will assume henceforth that this condition is satisfied.

\section{Asymptotic analysis}

This section is devoted to the behavior of $\boldsymbol{u}^{\epsilon}$, the unique solution of (16), when $\epsilon$ goes to 0 . For that we use a formal double-scale asymptotic method like in [Abdelmoula and Marigo 2000; Allaire 1992; Bensoussan et al. 1978; David et al. 2012; Marigo and Pideri 2011]. The goal is not to obtain rigorous results of convergence, but simply to formally construct the "limit" problem.

3.1. The assumed asymptotic expansion of $\boldsymbol{u}^{\epsilon}$. By virtue of the unidirectional character of the fibers, one can choose a two-dimensional domain $\boldsymbol{V}$ as the rescaled periodic cell characterizing the spatial distribution of the fibers; see [Bouchelaghem et al. 2007; Léné 1984; Marigo and Pideri 2011]. The fiber part and the matrix part of this cell are, respectively, the open sets $\boldsymbol{V}_{f}$ and $\boldsymbol{V}_{m}$ of the $\left(y_{1}, y_{2}\right)$ plane, while the interface is $\boldsymbol{I}=\partial \boldsymbol{V}_{f} \cap \partial \boldsymbol{V}_{m}$. Accordingly, one has

$$
\boldsymbol{V}=\boldsymbol{V}_{f} \cup \boldsymbol{I} \cup \boldsymbol{V}_{m}
$$


Moreover, the rigidity tensor and the mass density fields can be read as

$$
\begin{aligned}
& \mathrm{A}^{\epsilon}(\boldsymbol{x})=\mathrm{A}\left(\frac{\boldsymbol{x}^{\prime}}{\epsilon}\right) \quad \text { with } \mathrm{A}(\boldsymbol{y})= \begin{cases}\mathrm{A}^{f} & \text { if } \boldsymbol{y} \in \boldsymbol{V}_{f}, \\
\mathrm{~A}^{m} & \text { if } \boldsymbol{y} \in \boldsymbol{V}_{m},\end{cases} \\
& \rho^{\epsilon}(\boldsymbol{x})=\rho\left(\frac{\boldsymbol{x}^{\prime}}{\epsilon}\right) \quad \text { with } \rho(\boldsymbol{y})= \begin{cases}\rho_{f} & \text { if } \boldsymbol{y} \in \boldsymbol{V}_{f}, \\
\rho_{m} & \text { if } \boldsymbol{y} \in \boldsymbol{V}_{m} .\end{cases}
\end{aligned}
$$

This allows us to write problem (16) in the equivalent form

find $\boldsymbol{u}^{\epsilon} \in \mathscr{C}^{\epsilon} \quad$ such that $\int_{\Omega \backslash \boldsymbol{I}_{d}^{\epsilon}} \mathrm{A}^{\epsilon} \boldsymbol{\varepsilon}\left(\boldsymbol{u}^{\epsilon}\right) \cdot \boldsymbol{\varepsilon}(\boldsymbol{v}) d x=\int_{\Omega} \rho^{\epsilon} \boldsymbol{g} \cdot \boldsymbol{v} d x+\int_{\Gamma_{s}} \boldsymbol{F} \cdot \boldsymbol{v} d \Gamma$

$$
\text { for all } v \in \mathscr{C}^{\epsilon} \text {. }
$$

Following the classical two-scale procedure in homogenization theory of periodic media [Allaire 1992; Bensoussan et al. 1978], we assume that $\boldsymbol{u}^{\epsilon}$ can be expanded as follows:

$$
\boldsymbol{u}^{\epsilon}(\boldsymbol{x})=\sum_{i=0}^{\infty} \epsilon^{i} \boldsymbol{u}^{i}\left(\boldsymbol{x}, \frac{\boldsymbol{x}^{\prime}}{\epsilon}\right),
$$

where the fields $\boldsymbol{u}^{i}$ are defined in $\Omega \times V$ and $\boldsymbol{V}$-periodic (with respect to the microscopic variable $\boldsymbol{y}$ ). As far as their regularity with respect to $\boldsymbol{y}$ is concerned, one can discriminate according to whether $\boldsymbol{x}$ belongs to $\Omega_{c}$ or $\Omega_{d}$. Specifically, if $\boldsymbol{x} \in \Omega_{c}$, then $\boldsymbol{u}^{i}(\boldsymbol{x}, \cdot)$ must be continuous across $\boldsymbol{I}$, while if $\boldsymbol{x} \in \Omega_{d}$, then $u_{n}^{i}(\boldsymbol{x}, \cdot)$ only must be continuous across $\boldsymbol{I}$.

Using the chain rule, the strain field admits the expansion

$$
\boldsymbol{\varepsilon}\left(\boldsymbol{u}^{\epsilon}\right)(\boldsymbol{x})=\sum_{i=-1}^{\infty} \epsilon^{i}\left(\boldsymbol{\varepsilon}_{\boldsymbol{y}}\left(\boldsymbol{u}^{i+1}\right)\left(\boldsymbol{x}, \frac{\boldsymbol{x}^{\prime}}{\epsilon}\right)+\boldsymbol{\varepsilon}_{\boldsymbol{x}}\left(\boldsymbol{u}^{i}\right)\left(\boldsymbol{x}, \frac{\boldsymbol{x}^{\prime}}{\epsilon}\right)\right),
$$

where $\boldsymbol{\varepsilon}_{\boldsymbol{x}}(\boldsymbol{v})$ and $\boldsymbol{\varepsilon}_{\boldsymbol{y}}(\boldsymbol{v})$ denote, respectively, the symmetrized gradient of the displacement field $\boldsymbol{v}$ with respect to the macroscopic and microscopic coordinates; see (2)-(3).

3.2. Equations at various orders. Let us choose a two-scale smooth displacement field $\boldsymbol{v}^{\epsilon}(\boldsymbol{x})=\boldsymbol{v}\left(\boldsymbol{x}, \boldsymbol{x}^{\prime} / \epsilon\right), \boldsymbol{V}$-periodic and such that $\boldsymbol{v}(\boldsymbol{x}, \boldsymbol{y})=\mathbf{0}$ when $\boldsymbol{x} \in \Gamma_{c}$, as an element of $\mathscr{C}^{\epsilon}$ and let us insert it into (21) as the test field. After inserting the asymptotic expansion of $\boldsymbol{u}^{\epsilon}$ into (21) and identifying the terms at the same power of $\epsilon$, one obtains a sequence of variational problems for the $\boldsymbol{u}^{i}$, the first three of which are given below. (One formally replaces simple integrals over $\Omega$ by multiple integrals over $\Omega \times \boldsymbol{V}$ in the spirit of the double-scale approach [Allaire 1992].)

(1) At order $\epsilon^{-2}$ :

$$
0=\int_{\Omega_{c}} \int_{V} \mathrm{~A} \boldsymbol{\varepsilon}_{\boldsymbol{y}}\left(\boldsymbol{u}^{0}\right) \cdot \boldsymbol{\varepsilon}_{\boldsymbol{y}}(\boldsymbol{v}) d y d x+\int_{\Omega_{d}} \int_{\boldsymbol{V} \backslash \boldsymbol{I}} \mathrm{A} \boldsymbol{\varepsilon}_{\boldsymbol{y}}\left(\boldsymbol{u}^{0}\right) \cdot \boldsymbol{\varepsilon}_{\boldsymbol{y}}(\boldsymbol{v}) d y d x .
$$


(2) At order $\epsilon^{-1}$ :

$$
\begin{array}{r}
0=\int_{\Omega_{c}} \int_{V} \mathrm{~A} \boldsymbol{\varepsilon}_{\boldsymbol{y}}\left(\boldsymbol{u}^{0}\right) \cdot \boldsymbol{\varepsilon}_{\boldsymbol{x}}(\boldsymbol{v}) d y d x+\int_{\Omega_{d}} \int_{\boldsymbol{V} \backslash \boldsymbol{I}} \mathrm{A} \boldsymbol{\varepsilon}_{\boldsymbol{y}}\left(\boldsymbol{u}^{0}\right) \cdot \boldsymbol{\varepsilon}_{\boldsymbol{x}}(\boldsymbol{v}) d y d x \\
\quad+\int_{\Omega_{c}} \int_{V} \mathrm{~A}\left(\boldsymbol{\varepsilon}_{\boldsymbol{y}}\left(\boldsymbol{u}^{1}\right)+\boldsymbol{\varepsilon}_{\boldsymbol{x}}\left(\boldsymbol{u}^{0}\right)\right) \cdot \boldsymbol{\varepsilon}_{\boldsymbol{y}}(\boldsymbol{v}) d y d x \\
\quad+\int_{\Omega_{d}} \int_{\boldsymbol{V} \backslash \boldsymbol{I}} \mathrm{A}\left(\boldsymbol{\varepsilon}_{\boldsymbol{y}}\left(\boldsymbol{u}^{1}\right)+\boldsymbol{\varepsilon}_{\boldsymbol{x}}\left(\boldsymbol{u}^{0}\right)\right) \cdot \boldsymbol{\varepsilon}_{\boldsymbol{y}}(\boldsymbol{v}) d y d x
\end{array}
$$

(3) At order $\epsilon^{0}$ :

$$
\begin{array}{r}
\int_{\Omega_{c}} \int_{V} \mathrm{~A}\left(\boldsymbol{\varepsilon}_{\boldsymbol{y}}\left(\boldsymbol{u}^{2}\right)+\boldsymbol{\varepsilon}_{\boldsymbol{x}}\left(\boldsymbol{u}^{1}\right)\right) \cdot \boldsymbol{\varepsilon}_{\boldsymbol{y}}(\boldsymbol{v}) d y d x+\int_{\Omega_{d}} \int_{\boldsymbol{V} \backslash \boldsymbol{I}} \mathrm{A}\left(\boldsymbol{\varepsilon}_{\boldsymbol{y}}\left(\boldsymbol{u}^{2}\right)+\boldsymbol{\varepsilon}_{\boldsymbol{x}}\left(\boldsymbol{u}^{1}\right)\right) \cdot \boldsymbol{\varepsilon}_{\boldsymbol{y}}(\boldsymbol{v}) d y d x \\
+\int_{\Omega_{c}} \int_{V} \mathrm{~A}\left(\boldsymbol{\varepsilon}_{\boldsymbol{y}}\left(\boldsymbol{u}^{1}\right)+\boldsymbol{\varepsilon}_{\boldsymbol{x}}\left(\boldsymbol{u}^{0}\right)\right) \cdot \boldsymbol{\varepsilon}_{\boldsymbol{x}}(\boldsymbol{v}) d y d x+\int_{\Omega_{d}} \int_{V \backslash \boldsymbol{I}} \mathrm{A}\left(\boldsymbol{\varepsilon}_{\boldsymbol{y}}\left(\boldsymbol{u}^{1}\right)+\boldsymbol{\varepsilon}_{\boldsymbol{x}}\left(\boldsymbol{u}^{0}\right)\right) \cdot \boldsymbol{\varepsilon}_{\boldsymbol{x}}(\boldsymbol{v}) d y d x \\
=\int_{\Omega} \int_{V} \rho \boldsymbol{g} \cdot \boldsymbol{v} d y d x+\int_{\Gamma_{s}} \int_{V} \boldsymbol{F} \cdot \boldsymbol{v} d y d \Gamma .
\end{array}
$$

In (24)-(26), A and $\rho$ stand for the $\boldsymbol{V}$-periodic functions of $\boldsymbol{y}$ introduced in (19) and (20). Moreover, these variational equalities must hold for any smooth $\boldsymbol{v}(\boldsymbol{x}, \boldsymbol{y})$ which vanishes when $\boldsymbol{x} \in \Gamma_{c}$ as a function of $\boldsymbol{x}$, which is $\boldsymbol{V}$-periodic in $\boldsymbol{y}$, continuous across $\boldsymbol{I}$ when $\boldsymbol{x} \in \Omega_{c}$ and whose normal component $v_{n}$ is continuous across $\boldsymbol{I}$ when $\boldsymbol{x} \in \Omega_{d}$.

3.3. The form of $\boldsymbol{u}^{\mathbf{0}}$. By choosing $\boldsymbol{v}=\boldsymbol{u}^{0}$ in (24) (which is licit) and owing to the positivity of the elasticity tensors $\mathrm{A}^{f}$ and $\mathrm{A}^{m}$, one deduces that

$$
\boldsymbol{\varepsilon}_{\boldsymbol{y}}\left(\boldsymbol{u}^{0}\right)=\mathbf{0} \quad \text { in } \Omega_{c} \times \boldsymbol{V} \text { and in } \Omega_{d} \times(\boldsymbol{V} \backslash \boldsymbol{I}) .
$$

Let us discriminate the case when $x \in \Omega_{c}$ and that when $x \in \Omega_{d}$.

(1) When $\boldsymbol{x} \in \Omega_{c}$, since $\boldsymbol{\varepsilon}\left(\boldsymbol{u}^{0}\right)(\boldsymbol{x}, \boldsymbol{y})=\mathbf{0}$ for all $\boldsymbol{y} \in V, \boldsymbol{u}^{0}$ must be a rigid displacement with respect to $\boldsymbol{y}$. Recalling that $\boldsymbol{u}^{0}(\boldsymbol{x}, \boldsymbol{y}) \in \mathbb{R}^{3}$ and that $\boldsymbol{y}=\left(y_{1}, y_{2}\right)$, using (3) leads to

$$
\boldsymbol{u}^{0}(\boldsymbol{x}, \boldsymbol{y})=\boldsymbol{u}(\boldsymbol{x})+\omega(\boldsymbol{x}) \boldsymbol{e}_{3} \wedge \boldsymbol{y} \text { for all } \boldsymbol{y} \in \boldsymbol{V},
$$

where $\boldsymbol{u}(\boldsymbol{x}) \in \mathbb{R}^{3}$ and $\omega(\boldsymbol{x}) \in \mathbb{R}$. (Note that the rotations of axes $\boldsymbol{e}_{1}$ and $\boldsymbol{e}_{2}$ are automatically eliminated because $\boldsymbol{u}^{0}$ is independent of $y_{3}$.) But since $\boldsymbol{u}^{0}$ must be $\boldsymbol{V}$-periodic, one gets also $\omega(\boldsymbol{x})=0$. Finally, we have obtained that

$$
\text { for } \boldsymbol{x} \in \Omega_{c}: \quad \boldsymbol{u}^{0}(\boldsymbol{x}, \boldsymbol{y})=\boldsymbol{u}(\boldsymbol{x}) \quad \text { for all } \boldsymbol{y} \in \boldsymbol{V} .
$$

This result is the classical property of the homogenization theory which states that the leading term of the asymptotic displacement field expansion does not depend on the microscopic coordinates. However, this property holds true only because the fiber is perfectly bonded to the matrix, as we will see hereafter. 
(2) When $\boldsymbol{x} \in \Omega_{d}$, one has separately $\boldsymbol{\varepsilon}_{\boldsymbol{y}}\left(\boldsymbol{u}^{0}\right)(\boldsymbol{x}, \cdot)=\mathbf{0}$ in $\boldsymbol{V}_{f}$ and in $\boldsymbol{V}_{m}$. Therefore, $\boldsymbol{u}^{0}(\boldsymbol{x}, \boldsymbol{y})$ must be a rigid displacement field with respect to $\boldsymbol{y}$ in the matrix part $\boldsymbol{V}_{m}$ and a priori another rigid displacement field in the fiber part $\boldsymbol{V}_{f}$ of the cell $\boldsymbol{V}$. Accordingly, $\boldsymbol{u}^{0}(\boldsymbol{x}, \boldsymbol{y})$ must read as

$$
\boldsymbol{u}^{0}(\boldsymbol{x}, \boldsymbol{y})= \begin{cases}\boldsymbol{u}_{m}(\boldsymbol{x})+\omega_{m}(\boldsymbol{x}) \boldsymbol{e}_{3} \wedge \boldsymbol{y} & \text { for all } \boldsymbol{y} \in \boldsymbol{V}_{m}, \\ \boldsymbol{u}_{f}(\boldsymbol{x})+\omega_{f}(\boldsymbol{x}) \boldsymbol{e}_{3} \wedge \boldsymbol{y} & \text { for all } \boldsymbol{y} \in \boldsymbol{V}_{f},\end{cases}
$$

where $\boldsymbol{u}_{m}(\boldsymbol{x})$ and $\boldsymbol{u}_{f}(\boldsymbol{x})$ are in $\mathbb{R}^{3}, \omega_{m}(\boldsymbol{x})$ and $\omega_{f}(\boldsymbol{x})$ are in $\mathbb{R}$. Since $\boldsymbol{u}^{0}$ must be $\boldsymbol{V}$-periodic, one still gets $\omega_{m}(\boldsymbol{x})=0$. Let us write now the continuity of $u_{n}^{0}$ across $\boldsymbol{I}$. We can take the center of the (circular) fiber cross-section as the origin of the $\left(y_{1}, y_{2}\right)$ plane without loss of generality. Accordingly, $\boldsymbol{n}=\boldsymbol{y} / R=\cos \theta \boldsymbol{e}_{1}+\sin \theta \boldsymbol{e}_{2}$ for $\boldsymbol{y} \in \boldsymbol{I}$. Therefore, $\llbracket \boldsymbol{u}^{0} \rrbracket \cdot \boldsymbol{n}=0$ on $\boldsymbol{I}$ reads as

$$
\cos \theta\left(\boldsymbol{u}_{m}(\boldsymbol{x})-\boldsymbol{u}_{f}(\boldsymbol{x})\right) \cdot \boldsymbol{e}_{1}+\sin \theta\left(\boldsymbol{u}_{m}(\boldsymbol{x})-\boldsymbol{u}_{f}(\boldsymbol{x})\right) \cdot \boldsymbol{e}_{2}=0 \quad \text { for all } \theta \in[0,2 \pi],
$$

from which one immediately deduces that $\boldsymbol{u}_{f}(\boldsymbol{x})=\boldsymbol{u}_{m}(\boldsymbol{x})+\delta(\boldsymbol{x}) \boldsymbol{e}_{3}$. Finally, we have obtained that

$$
\text { for } \boldsymbol{x} \in \Omega_{d}: \quad \boldsymbol{u}^{0}(\boldsymbol{x}, \boldsymbol{y})=\left\{\begin{array}{cl}
\boldsymbol{u}(\boldsymbol{x}) & \text { for all } \boldsymbol{y} \in \boldsymbol{V}_{m}, \\
\boldsymbol{u}(\boldsymbol{x})+\delta(\boldsymbol{x}) \boldsymbol{e}_{3}+\omega(\boldsymbol{x}) \boldsymbol{e}_{3} \wedge \boldsymbol{y} & \text { for all } \boldsymbol{y} \in \boldsymbol{V}_{f} .
\end{array}\right.
$$

For future reference, let us denote by $\mathscr{R}_{d}$ the set of the $V$-periodic displacement fields $\boldsymbol{w}$ such that $\boldsymbol{\varepsilon}_{\boldsymbol{y}}(\boldsymbol{w})=0$ in $\boldsymbol{V} \backslash \boldsymbol{I}$ and $\llbracket w_{n} \rrbracket=0$ on $\boldsymbol{I}$; i.e.,

$$
\mathscr{R}_{d}=\left\{\boldsymbol{w}: \boldsymbol{w}(\boldsymbol{y})=\left\{\begin{array}{cl}
\boldsymbol{a} & \text { for } \boldsymbol{y} \in \boldsymbol{V}_{m}, \\
\boldsymbol{a}+\delta \boldsymbol{e}_{3}+\omega \boldsymbol{e}_{3} \wedge \boldsymbol{y} & \text { for } \boldsymbol{y} \in \boldsymbol{V}_{f},
\end{array} \boldsymbol{a} \in \mathbb{R}^{3}, \delta \in \mathbb{R}, \omega \in \mathbb{R}\right\} .\right.
$$

Thus $\boldsymbol{u}^{0}(\boldsymbol{x}, \cdot) \in \mathscr{R}_{d}$ when $\boldsymbol{x} \in \Omega_{d}$. This result differs from the usual property of the homogenization theory. Indeed, because of the debonding of the fiber from the matrix, the leading term of the asymptotic displacement field expansion depends here on the microscopic coordinates. Moreover, two new macroscopic scalar fields appear in the effective kinematics of the composite. Specifically, the vector field $\boldsymbol{u}$ represents the macroscopic displacement of the matrix while the scalar fields $\delta$ and $\omega$ represent the longitudinal sliding and the relative rotation of the fibers with respect to the matrix. We have obtained a generalized continuous medium.

Let us summarize all results obtained in this subsection:

Proposition 2. The first-order displacement $\boldsymbol{u}^{0}(\boldsymbol{x}, \boldsymbol{y})$ takes two different forms according to whether $\boldsymbol{x}$ is in $\Omega_{c}$ or in $\Omega_{d}$. Specifically,

$$
\begin{aligned}
& \text { for } \boldsymbol{x} \in \Omega_{c}: \quad \boldsymbol{u}^{0}(\boldsymbol{x}, \boldsymbol{y})=\boldsymbol{u}(\boldsymbol{x}) \text { for all } \boldsymbol{y} \in \boldsymbol{V}, \\
& \text { for } \boldsymbol{x} \in \Omega_{d}: \quad \boldsymbol{u}^{0}(\boldsymbol{x}, \boldsymbol{y})=\left\{\begin{array}{cl}
\boldsymbol{u}(\boldsymbol{x}) & \text { for all } \boldsymbol{y} \in \boldsymbol{V}_{m}, \\
\boldsymbol{u}(\boldsymbol{x})+\delta(\boldsymbol{x}) \boldsymbol{e}_{3}+\omega(\boldsymbol{x}) \boldsymbol{e}_{3} \wedge \boldsymbol{y} & \text { for all } \boldsymbol{y} \in \boldsymbol{V}_{f} .
\end{array}\right.
\end{aligned}
$$

Therefore, the effective kinematic behavior in the debonded part of the composite 
body is that of a generalized continuous medium where appear the sliding and the rotation of the fibers with respect to the matrix.

Remark 2. The macroscopic displacement fields $\boldsymbol{u}, \delta$ and $\omega$ can be defined in the whole domain $\Omega$ but $\delta$ and $\omega$ must vanish in $\Omega_{c}$. Moreover, those fields have to be sufficiently smooth in order that the effective elastic energy be finite. Their smoothness will be specified once the effective behavior is obtained. In the same way, the boundary conditions that $\boldsymbol{u}, \delta$ and $\omega$ have to satisfy on $\Gamma_{c}$ will be specified later.

3.4. The elementary cell problems. Inserting (27) and (28) into (25) leads to

$$
\begin{aligned}
0=\int_{\Omega_{c}} & \int_{V} \mathrm{~A}\left(\boldsymbol{\varepsilon}_{\boldsymbol{y}}\left(\boldsymbol{u}^{1}\right)+\boldsymbol{\varepsilon}(\boldsymbol{u})\right) \cdot \boldsymbol{\varepsilon}_{\boldsymbol{y}}(\boldsymbol{v}) d y d x \\
& +\int_{\Omega_{d}} \int_{\boldsymbol{V} \backslash \boldsymbol{I}} \mathrm{A}\left(\boldsymbol{\varepsilon}_{\boldsymbol{y}}\left(\boldsymbol{u}^{1}\right)+\boldsymbol{\varepsilon}(\boldsymbol{u})+\boldsymbol{\varepsilon}\left(\delta \boldsymbol{e}_{3}\right)+\boldsymbol{\varepsilon}_{\boldsymbol{x}}\left(\omega \boldsymbol{e}_{3} \wedge \boldsymbol{y}\right)\right) \cdot \boldsymbol{\varepsilon}_{\boldsymbol{y}}(\boldsymbol{v}) d y d x .
\end{aligned}
$$

Assuming at this stage that the fields $\boldsymbol{u}, \delta$ and $\omega$ are known, (30) will allow us to determine $\boldsymbol{u}^{1}$ in terms of the gradient of $\boldsymbol{u}, \delta$ and $\omega$. For that, we have still to discriminate between the domains $\Omega_{c}$ and $\Omega_{d}$.

(1) Let us first choose $\boldsymbol{v}$ such that $\boldsymbol{v}(\boldsymbol{x}, \boldsymbol{y})=\varphi(\boldsymbol{x}) \boldsymbol{w}(\boldsymbol{y})$ with $\varphi \in \mathscr{D}\left(\Omega_{c}\right)$ (the set of indefinitely differentiable functions with compact support in $\Omega_{c}$ ) and $\boldsymbol{w} \in \mathscr{H}_{c}$, where $\mathscr{H}_{c}$ denotes the Hilbert space of vector fields which are $\boldsymbol{V}$-periodic and whose components are in $H^{1}(\boldsymbol{V})$; i.e.,

$$
\mathscr{H}_{c}=\left\{\boldsymbol{w} \in H^{1}\left(\boldsymbol{V} ; \mathbb{R}^{3}\right): \boldsymbol{w} \text { is } \boldsymbol{V} \text {-periodic }\right\} .
$$

Then (30) becomes: at almost all $\boldsymbol{x} \in \Omega_{c}$ and for all $\boldsymbol{w} \in \mathscr{H}_{c}$,

$$
\int_{V} \mathrm{~A}(\boldsymbol{y}) \boldsymbol{\varepsilon}_{\boldsymbol{y}}\left(\boldsymbol{u}^{1}\right)(\boldsymbol{x}, \boldsymbol{y}) \cdot \boldsymbol{\varepsilon}(\boldsymbol{w})(\boldsymbol{y}) d y+\boldsymbol{\varepsilon}(\boldsymbol{u})(\boldsymbol{x}) \cdot \int_{V} \mathrm{~A}(\boldsymbol{y}) \boldsymbol{\varepsilon}(\boldsymbol{w})(\boldsymbol{y}) d y=0 .
$$

Hence, by linearity, $\boldsymbol{u}^{1}$ can read as

$$
\text { for } \boldsymbol{x} \in \Omega_{c}: \quad u_{k}^{1}(\boldsymbol{x}, \boldsymbol{y})=\varepsilon(\boldsymbol{u})_{i j}(\boldsymbol{x}) \chi_{k}^{i j}(\boldsymbol{y})+\bar{u}_{k}(\boldsymbol{x}) \text { for all } \boldsymbol{y} \in \boldsymbol{V},
$$

where, for $i, j \in\{1,2,3\}$, the vector fields $\chi^{i j}$ are the elements of $\mathscr{H}_{c}$ solving the so-called cell problems

$$
\int_{V} \mathrm{~A}_{p q r s} \varepsilon\left(\chi^{i j}\right)_{p q} \varepsilon(\boldsymbol{w})_{r s} d y+\int_{V} \mathrm{~A}_{i j r s} \varepsilon(\boldsymbol{w})_{r s} d y=0 \quad \text { for all } \boldsymbol{w} \in \mathscr{H}_{c} .
$$

In (31), $\overline{\boldsymbol{u}}(\boldsymbol{x})$ remains undetermined at this stage.

(2) Let us now choose $\boldsymbol{v}$ such that $\boldsymbol{v}(\boldsymbol{x}, \boldsymbol{y})=\varphi(\boldsymbol{x}) \boldsymbol{w}(\boldsymbol{y})$ with $\varphi \in \mathscr{D}\left(\Omega_{d}\right)$ and $\boldsymbol{w} \in \mathscr{H}_{d}$, where

$$
\mathscr{H}_{d}=\left\{\boldsymbol{w} \in H^{1}\left(\boldsymbol{V} \backslash \boldsymbol{I} ; \mathbb{R}^{3}\right): \boldsymbol{w} \text { is } \boldsymbol{V} \text {-periodic, } \llbracket w_{n} \rrbracket=0 \text { on } \boldsymbol{I}\right\} .
$$


Then (30) becomes: at almost all $\boldsymbol{x} \in \Omega_{d}$ and for all $\boldsymbol{w} \in \mathscr{H}_{d}$,

$$
\begin{aligned}
0=\int_{\boldsymbol{V} \backslash \boldsymbol{I}} \mathrm{A}(\boldsymbol{y}) \boldsymbol{\varepsilon}_{\boldsymbol{y}}\left(\boldsymbol{u}^{1}\right)(\boldsymbol{x}, \boldsymbol{y}) \cdot \boldsymbol{\varepsilon}(\boldsymbol{w})(\boldsymbol{y}) d y \\
\quad+\boldsymbol{\varepsilon}(\boldsymbol{u})(\boldsymbol{x}) \cdot \int_{\boldsymbol{V} \backslash \boldsymbol{I}} \mathrm{A}(\boldsymbol{y}) \boldsymbol{\varepsilon}(\boldsymbol{w})(\boldsymbol{y}) d y+\boldsymbol{\varepsilon}\left(\delta \boldsymbol{e}_{3}\right)(\boldsymbol{x}) \cdot \int_{V_{f}} \mathrm{~A}^{f} \boldsymbol{\varepsilon}(\boldsymbol{w})(\boldsymbol{y}) d y \\
\quad+\boldsymbol{\varepsilon}\left(\omega \boldsymbol{e}_{2}\right)(\boldsymbol{x}) \cdot \int_{\boldsymbol{V}_{f}} y_{1} \mathrm{~A}^{f} \boldsymbol{\varepsilon}(\boldsymbol{w})(\boldsymbol{y}) d y-\boldsymbol{\varepsilon}\left(\omega \boldsymbol{e}_{1}\right)(\boldsymbol{x}) \cdot \int_{V_{f}} y_{2} \mathrm{~A}^{f} \boldsymbol{\varepsilon}(\boldsymbol{w})(\boldsymbol{y}) d y .
\end{aligned}
$$

Hence, by linearity, $\boldsymbol{u}^{1}$ can read as

for $\boldsymbol{x} \in \Omega_{d}: \quad \boldsymbol{u}^{1}(\boldsymbol{x}, \boldsymbol{y})=\varepsilon(\boldsymbol{u})_{i j}(\boldsymbol{x}) \xi^{i j}(\boldsymbol{y})+\frac{\partial \delta}{\partial x_{i}}(\boldsymbol{x}) \boldsymbol{D}^{i}(\boldsymbol{y})+\frac{\partial \omega}{\partial x_{i}}(\boldsymbol{x}) \boldsymbol{W}^{i}(\boldsymbol{y})+\overline{\boldsymbol{u}}(\boldsymbol{x}, \boldsymbol{y})$$$
\text { for all } \boldsymbol{y} \in \boldsymbol{V} \backslash \boldsymbol{I} \text {, }
$$

where $\overline{\boldsymbol{u}}(\boldsymbol{x}, \cdot)$ is an element of $\mathscr{R}_{d}$ that remains undetermined at this stage, and the vector fields $\boldsymbol{\xi}^{i j}, \boldsymbol{D}^{i}$ and $\boldsymbol{W}^{i}$, for $i, j \in\{1,2,3\}$, are the elements of $\mathscr{H}_{d}$ solving the following new cell problems:

$$
\begin{array}{r}
\int_{\boldsymbol{V} \backslash \boldsymbol{I}} \mathrm{A}_{p q r s} \varepsilon\left(\boldsymbol{\xi}^{i j}\right)_{p q} \varepsilon(\boldsymbol{w})_{r s} d y+\int_{\boldsymbol{V} \backslash \boldsymbol{I}} \mathrm{A}_{i j r s} \varepsilon(\boldsymbol{w})_{r s} d y=0, \\
\int_{\boldsymbol{V} \backslash \boldsymbol{I}} \mathrm{A}_{p q r s} \varepsilon\left(\boldsymbol{D}^{i}\right)_{p q} \varepsilon(\boldsymbol{w})_{r s} d y+\int_{\boldsymbol{V}_{f}} \mathrm{~A}_{3 i r s}^{f} \varepsilon(\boldsymbol{w})_{r s} d y=0, \\
\int_{\boldsymbol{V} \backslash \boldsymbol{I}} \mathrm{A}_{p q r s} \varepsilon\left(\boldsymbol{W}^{i}\right)_{p q} \varepsilon(\boldsymbol{w})_{r s} d y+\int_{\boldsymbol{V}_{f}}\left(\boldsymbol{e}_{3} \wedge \boldsymbol{y}\right) \cdot \boldsymbol{e}_{q} \mathrm{~A}_{i q r s}^{f} \varepsilon(\boldsymbol{w})_{r s} d y=0 .
\end{array}
$$

In (34)-(36) equality holds for all $\boldsymbol{w} \in \mathscr{H}_{d}$.

Let us study each of these cell problems.

- Each $\chi^{i j}$ is uniquely determined up to a translation which can be fixed by imposing that $\int_{V} \chi^{i j} d y=\mathbf{0}$. It corresponds to the microscopic response of the representative volume element submitted to the macroscopic strain tensor $\boldsymbol{e}_{i} \otimes_{s} \boldsymbol{e}_{j}$. In other words, the $\chi^{i j}$ are given by the classical microscopic problems appearing in the homogenization theory [Allaire 1992; Bensoussan et al. 1978]. By virtue of the symmetries of the rigidity tensors $\mathrm{A}^{f}$ and $\mathrm{A}^{m}$, one has $\chi^{i j}=\chi^{j i}$ and hence there exist exactly six independent cell problems. Since the periodicity is twodimensional and since the fibers and the matrix are isotropic, all the $\chi^{i j}$ enjoy some general properties. For instance,

$$
\chi_{3}^{\alpha \beta}=\chi_{3}^{33}=\chi_{\beta}^{\alpha 3}=0 \quad \text { for all } \alpha, \beta \in\{1,2\} .
$$

Additional symmetry properties appear when the cell itself enjoys additional symmetries [Léné 1984]. The practical determination of the $\chi^{i j}$ requires some numerical computation. 
- All preceding comments on the $\chi^{i j}$ remain true for the $\xi^{i j}$ (except that $\xi^{i j}$ is uniquely determined up to an element of $\mathscr{R}_{d}$ ). Note however that $\xi^{i j}$ differs (in general) from $\chi^{i j}$ because of the possibility of a tangential discontinuity of $\xi^{i j}$ on $\boldsymbol{I}$. A consequence of this additional degree of freedom is that the shear stress associated with $\xi^{i j}$ necessarily vanishes on $\boldsymbol{I}$ while this is not in general the case for $\chi^{i j}$.

- The fields $\boldsymbol{D}^{1}$ and $\boldsymbol{D}^{2}$ can be obtained in a closed form. Specifically, one gets

$$
\text { for } \alpha \in\{1,2\}: \quad \boldsymbol{D}^{\alpha}(y)=\left\{\begin{array}{cl}
\mathbf{0}, & \boldsymbol{y} \in \boldsymbol{V}_{m}, \\
-y_{\alpha} \boldsymbol{e}_{3}, & \boldsymbol{y} \in \boldsymbol{V}_{f},
\end{array} \quad+\text { an arbitrary element of } \mathscr{R}_{d} .\right.
$$

The verification is straightforward and left to the reader. On the other hand, $\boldsymbol{D}^{3}$ cannot be obtained in a closed form (except if $\lambda_{f}=0$ ) but can be simplified. Indeed, as for the $\xi^{i j}$, by virtue of the isotropy of the fibers and the matrix, one gets that $D_{3}^{3}=0$ and finally the problem for $\boldsymbol{D}^{3}$ can read as

$$
\begin{aligned}
& \int_{\boldsymbol{V} \backslash \boldsymbol{I}} \lambda \varepsilon\left(\boldsymbol{D}^{3}\right)_{\alpha \alpha} \varepsilon(\boldsymbol{w})_{\beta \beta}+2 \mu \varepsilon\left(\boldsymbol{D}^{3}\right)_{\alpha \beta} \varepsilon(\boldsymbol{w})_{\alpha \beta} d y+\int_{\boldsymbol{V}_{f}} \lambda_{f} \varepsilon(\boldsymbol{w})_{\beta \beta} d y=0 \\
& \text { for all } \boldsymbol{w} \in \mathscr{H}_{d} \text {. }
\end{aligned}
$$

It corresponds to the response of the cell when the fiber is submitted to a macroscopic longitudinal stretching $\boldsymbol{e}_{3} \otimes \boldsymbol{e}_{3}$ while the matrix is macroscopically unstrained. That response is not trivial because of the contact between the fiber and the matrix. This contact implies the existence of a normal stress $\sigma_{n n}$ at the interface $\boldsymbol{I}$ which induces a deformation of the matrix.

- All the fields $\boldsymbol{W}^{i}$ can be obtained in a closed form. Let us first show that

$$
\boldsymbol{W}^{3} \in \mathscr{R}_{d} .
$$

Indeed, the integral over $\boldsymbol{V}_{f}$ in (36) for $i=3$ vanishes as proved below:

$$
\begin{aligned}
\int_{V_{f}}\left(\boldsymbol{e}_{3} \wedge \boldsymbol{y}\right) \cdot \boldsymbol{e}_{\beta} \mathrm{A}_{3 \beta k l}^{f} \varepsilon(\boldsymbol{w})_{k l} d y & =\int_{V_{f}} \mu_{f}\left(\boldsymbol{e}_{3} \wedge \boldsymbol{y}\right) \cdot \boldsymbol{e}_{\beta} \frac{\partial w_{3}}{\partial y_{\beta}} d y \\
& =-\int_{V_{f}} \mu_{f}\left(\boldsymbol{e}_{3} \wedge \boldsymbol{e}_{\beta}\right) \cdot \boldsymbol{e}_{\beta} w_{3} d y+\int_{\boldsymbol{I}} \mu_{f}\left(\boldsymbol{e}_{3} \wedge \boldsymbol{y}\right) \cdot \boldsymbol{n} w_{3} d s \\
& =0 .
\end{aligned}
$$

The last equality above is due to the fact that $\boldsymbol{n}=\boldsymbol{y} / R$ on $\boldsymbol{I}$. Inserting this property and taking $\boldsymbol{w}=\boldsymbol{W}^{3}$ in (36) for $i=3$ leads to

$$
\int_{\boldsymbol{V} \backslash \boldsymbol{I}} \mathrm{A} \boldsymbol{\varepsilon}\left(\boldsymbol{W}^{3}\right) \cdot \boldsymbol{\varepsilon}\left(\boldsymbol{W}^{3}\right) d y=0 .
$$

Therefore $\boldsymbol{\varepsilon}\left(\boldsymbol{W}^{3}\right)=\mathbf{0}$ which is the desired result. Since the undetermined element of $\mathscr{R}_{d}$ does not play any role, one can consider that $\boldsymbol{W}^{3}=\mathbf{0}$. Note that this property 
holds true because the fiber has a circular section and is isotropic.

Let us now verify that $\boldsymbol{W}^{1}$ and $\boldsymbol{W}^{2}$ are given by for $\alpha \in\{1,2\}: \boldsymbol{W}^{\alpha}(y)=\left\{\begin{array}{cl}\mathbf{0}, & \boldsymbol{y} \in \boldsymbol{V}_{m}, \\ -y_{\alpha} \boldsymbol{e}_{3} \wedge \boldsymbol{y}, & \boldsymbol{y} \in \boldsymbol{V}_{f},\end{array} \quad+\right.$ an arbitrary element of $\mathscr{R}_{d}$. (40) Let us first remark that $\llbracket \boldsymbol{W}^{\alpha} \rrbracket \cdot \boldsymbol{n}=0$ on $\boldsymbol{I}$ because $\left(\boldsymbol{e}_{3} \wedge \boldsymbol{y}\right) \cdot \boldsymbol{n}=0$. Hence $\boldsymbol{W}^{\alpha} \in \mathscr{H}_{d}$. Let us now calculate the strain field $\boldsymbol{\varepsilon}\left(\boldsymbol{W}^{\alpha}\right)$ for $\alpha \in\{1,2\}$ :

$$
2 \varepsilon\left(\boldsymbol{W}^{\alpha}\right)_{p q}=-\left(\boldsymbol{e}_{3} \wedge \boldsymbol{y}\right) \cdot \boldsymbol{e}_{p} \delta_{\alpha q}-\left(\boldsymbol{e}_{3} \wedge \boldsymbol{y}\right) \cdot \boldsymbol{e}_{q} \delta_{\alpha p} \quad \text { for all } p, q \in\{1,2,3\} .
$$

Therefore, one gets $\mathrm{A}_{p q r s}^{f} \varepsilon\left(\boldsymbol{W}^{\alpha}\right)_{p q}=-\left(\boldsymbol{e}_{3} \wedge \boldsymbol{y}\right) \cdot \boldsymbol{e}_{q} \mathrm{~A}_{\alpha q r s}^{f}$, from which one easily deduces that (36) is satisfied for $i=\alpha$.

3.5. The form of $\sigma^{0}$. The form of the leading term $\sigma^{0}$ of the stress field is obtained via the constitutive relations (8) and the strain expansion (23). Specifically, one gets

$$
\sigma^{0}(x, y)=\mathrm{A}(y)\left(\varepsilon_{x}\left(u^{0}\right)(x, y)+\varepsilon_{y}\left(u^{1}\right)(x, y)\right) .
$$

Let us discriminate once more between the domains $\Omega_{c}$ and $\Omega_{d}$ to obtain the stress field $\sigma^{0}$ in terms of the generalized strain fields $\boldsymbol{\varepsilon}(\boldsymbol{u}), \nabla \delta, \nabla \omega$ and of the microscopic strain fields associated with the solutions of the cell problems.

(1) For $x \in \Omega_{c}$. By virtue of (27) and (31), one gets

$$
\boldsymbol{\sigma}^{0}(\boldsymbol{x}, \boldsymbol{y})=\mathrm{A}(\boldsymbol{y})\left(\boldsymbol{\varepsilon}(\boldsymbol{u})(\boldsymbol{x})+\varepsilon(\boldsymbol{u})_{i j}(\boldsymbol{x}) \boldsymbol{\varepsilon}\left(\chi^{i j}\right)(\boldsymbol{y})\right),
$$

which is the usual expression of the stress distribution given by the homogenization theory. Of course, all cell problems give a contribution to that stress distribution.

(2) For $\boldsymbol{x} \in \Omega_{d}$. By virtue of (28) and (33), one gets, for all $\boldsymbol{y} \in \boldsymbol{V} \backslash \boldsymbol{I}$,

$$
\boldsymbol{\sigma}^{0}(\boldsymbol{x}, \boldsymbol{y})=\mathrm{A}(\boldsymbol{y})\left(\boldsymbol{\varepsilon}(\boldsymbol{u})(\boldsymbol{x})+\varepsilon(\boldsymbol{u})_{i j}(\boldsymbol{x}) \boldsymbol{\varepsilon}\left(\boldsymbol{\xi}^{i j}\right)(\boldsymbol{y})\right)+\frac{\partial \delta}{\partial x_{i}}(\boldsymbol{x}) \boldsymbol{S}^{i}(\boldsymbol{y})+\frac{\partial \omega}{\partial x_{i}}(\boldsymbol{x}) \boldsymbol{T}^{i}(\boldsymbol{y}),
$$

with

$$
\begin{aligned}
& S_{r s}^{i}(\boldsymbol{y})=\left\{\begin{array}{cl}
\mathrm{A}_{p q r s}^{m} \varepsilon\left(\boldsymbol{D}^{i}\right)_{p q}(\boldsymbol{y}) & \text { if } \boldsymbol{y} \in \boldsymbol{V}_{m}, \\
\mathrm{~A}_{p q r s}^{f} \varepsilon\left(\boldsymbol{D}^{i}\right)_{p q}(\boldsymbol{y})+\mathrm{A}_{3 i r s}^{f} & \text { if } \boldsymbol{y} \in \boldsymbol{V}_{f}
\end{array}\right. \\
& T_{r s}^{i}(\boldsymbol{y})=\left\{\begin{array}{cc}
\mathrm{A}_{p q r s}^{m} \varepsilon\left(\boldsymbol{W}^{i}\right)_{p q}(\boldsymbol{y}) & \text { if } \boldsymbol{y} \in \boldsymbol{V}_{m}, \\
\mathrm{~A}_{p q r s}^{f} \varepsilon\left(\boldsymbol{W}^{i}\right)_{p q}(\boldsymbol{y})+\mathrm{A}_{i q r s}^{f}\left(\boldsymbol{e}_{3} \wedge \boldsymbol{y}\right) \cdot \boldsymbol{e}_{q} & \text { if } \boldsymbol{y} \in \boldsymbol{V}_{f} .
\end{array}\right.
\end{aligned}
$$

Moreover, (37) gives $\boldsymbol{S}^{\alpha}=\mathbf{0}$ and (40) gives $\boldsymbol{T}^{\alpha}=\mathbf{0}$ for $\alpha \in\{1,2\}$. In other words the cell problems associated with $\partial \delta / \partial x_{\alpha}$ or with $\partial \omega / \partial x_{\alpha}$ induce no stress. Since $\boldsymbol{W}^{3}$ vanishes, $\boldsymbol{T}^{3}$ reads as

$$
\boldsymbol{T}^{3}(\boldsymbol{y})=\left\{\begin{array}{cl}
\mathbf{0} & \text { if } \boldsymbol{y} \in \boldsymbol{V}_{m}, \\
2 \mu_{f}\left(-y_{2} \boldsymbol{e}_{3} \otimes_{s} \boldsymbol{e}_{1}+y_{1} \boldsymbol{e}_{3} \otimes_{s} \boldsymbol{e}_{2}\right) & \text { if } \boldsymbol{y} \in \boldsymbol{V}_{f} .
\end{array}\right.
$$


Note that this stress distribution corresponds to that given by a torsion of a cylinder with a circular cross-section. The only nonzero component is the orthoradial one $\sigma_{3 \theta}$ which is proportional to $r$, the distance to the axis. Moreover, there is no interaction with the matrix.

On the other hand, $S^{3}$ cannot be obtained in a closed form, but can be simplified by using (38):

$$
\begin{aligned}
& S_{\alpha \beta}^{3}(\boldsymbol{y})=\left\{\begin{array}{cc}
\lambda_{m} \varepsilon_{\gamma \gamma}\left(\boldsymbol{D}^{3}\right)(\boldsymbol{y}) \delta_{\alpha \beta}+2 \mu_{m} \varepsilon_{\alpha \beta}\left(\boldsymbol{D}^{3}\right)(\boldsymbol{y}) & \text { if } \boldsymbol{y} \in \boldsymbol{V}_{m}, \\
\lambda_{f}\left(1+\varepsilon_{\gamma \gamma}\left(\boldsymbol{D}^{3}\right)(\boldsymbol{y})\right) \delta_{\alpha \beta}+2 \mu_{f} \varepsilon_{\alpha \beta}\left(\boldsymbol{D}^{3}\right)(\boldsymbol{y}) & \text { if } \boldsymbol{y} \in \boldsymbol{V}_{f},
\end{array}\right. \\
& S_{33}^{3}(\boldsymbol{y})=\left\{\begin{array}{cc}
\lambda_{m} \varepsilon_{\gamma \gamma}\left(\boldsymbol{D}^{3}\right)(\boldsymbol{y}) & \text { if } \boldsymbol{y} \in \boldsymbol{V}_{m}, \\
\lambda_{f}\left(1+\varepsilon_{\gamma \gamma}\left(\boldsymbol{D}^{3}\right)(\boldsymbol{y})\right)+2 \mu_{f} & \text { if } \boldsymbol{y} \in \boldsymbol{V}_{f},
\end{array}\right.
\end{aligned}
$$

and $S_{\alpha 3}^{3}=0$ in $\boldsymbol{V}_{f} \cup \boldsymbol{V}_{m}$. As it was already noted, there is an interaction between the fiber and the matrix because of the contact assumption.

Finally, $\boldsymbol{\sigma}^{0}(\boldsymbol{x}, \cdot)$ can read in $\boldsymbol{V} \backslash \boldsymbol{I}$ as

$\boldsymbol{\sigma}^{0}(\boldsymbol{x}, \boldsymbol{y})=\mathrm{A}(\boldsymbol{y})\left(\boldsymbol{\varepsilon}(\boldsymbol{u})(\boldsymbol{x})+\varepsilon(\boldsymbol{u})_{i j}(\boldsymbol{x}) \boldsymbol{\varepsilon}\left(\boldsymbol{\xi}^{i j}\right)(\boldsymbol{y})\right)+\frac{\partial \delta}{\partial x_{3}}(\boldsymbol{x}) \boldsymbol{S}^{3}(\boldsymbol{y})+\frac{\partial \omega}{\partial x_{3}}(\boldsymbol{x}) \boldsymbol{T}^{3}(\boldsymbol{y})$,

which includes the contribution of the longitudinal stretching and the torsion of the fibers.

3.6. The macroscopic problem. To obtain the problem which gives the macroscopic fields $\boldsymbol{u}, \delta$ and $\omega$, we choose a displacement field $\boldsymbol{v}$ in (26) of the same type as $\boldsymbol{u}^{0}$, i.e., such that $\boldsymbol{\varepsilon}_{\boldsymbol{y}}(\boldsymbol{v})=\mathbf{0}$. Specifically, one sets

$$
\boldsymbol{v}^{*}(\boldsymbol{x}, \boldsymbol{y})=\left\{\begin{array}{cl}
\boldsymbol{u}^{*}(\boldsymbol{x}) & \text { in }\left(\Omega_{c} \times V\right) \cup\left(\Omega_{d} \times \boldsymbol{V}_{m}\right), \\
\boldsymbol{u}^{*}(\boldsymbol{x})+\delta^{*}(\boldsymbol{x}) \boldsymbol{e}_{3}+\omega^{*}(\boldsymbol{x}) \boldsymbol{e}_{3} \wedge \boldsymbol{y} & \text { in } \Omega_{d} \times \boldsymbol{V}_{f}
\end{array}\right.
$$

and inserts such a $\boldsymbol{v}^{*}$ into (26). Then the terms in $\boldsymbol{\varepsilon}_{\boldsymbol{y}}\left(\boldsymbol{u}^{2}\right)+\boldsymbol{\varepsilon}_{\boldsymbol{x}}\left(\boldsymbol{u}^{1}\right)$ disappear because $\boldsymbol{\varepsilon}_{\boldsymbol{y}}(\boldsymbol{v})=0$. By virtue of (41), (26) becomes

$$
\begin{aligned}
& \int_{\Omega} \int_{V} \boldsymbol{\sigma}^{0}(\boldsymbol{x}, \boldsymbol{y}) \cdot \boldsymbol{\varepsilon}\left(\boldsymbol{u}^{*}\right)(\boldsymbol{x}) d y d x \\
& \quad+\int_{\Omega_{d}} \int_{V_{f}} \boldsymbol{\sigma}^{0}(\boldsymbol{x}, \boldsymbol{y}) \cdot\left(\boldsymbol{\varepsilon}\left(\delta^{*} \boldsymbol{e}_{3}\right)(\boldsymbol{x})+\boldsymbol{\varepsilon}\left(\omega^{*} \boldsymbol{e}_{3} \wedge \boldsymbol{e}_{\alpha}\right)(\boldsymbol{x}) y_{\alpha}\right) d y d x \\
& =\int_{\Omega} \int_{V} \rho(\boldsymbol{y}) \boldsymbol{g}(\boldsymbol{x}) \cdot \boldsymbol{u}^{*}(\boldsymbol{x}) d y d x+\int_{\Omega_{d}} \int_{V_{f}} \rho_{f}\left(g_{3}(\boldsymbol{x}) \delta^{*}(\boldsymbol{x})+\left(\boldsymbol{e}_{3} \wedge \boldsymbol{y}\right) \cdot \boldsymbol{g}(\boldsymbol{x}) \omega^{*}(\boldsymbol{x})\right) d y d x \\
& +\int_{\Gamma_{s}} \int_{\boldsymbol{V}} \boldsymbol{F}(\boldsymbol{x}) \cdot \boldsymbol{u}^{*}(\boldsymbol{x}) d y d \Gamma+\int_{\Gamma_{s}} \int_{V_{f}}\left(F_{3}(\boldsymbol{x}) \delta^{*}(\boldsymbol{x})+\left(\boldsymbol{e}_{3} \wedge \boldsymbol{y}\right) \cdot \boldsymbol{F}(\boldsymbol{x}) \omega^{*}(\boldsymbol{x})\right) d y d \Gamma .
\end{aligned}
$$

Let us denote by $\langle\varphi\rangle$ the mean value of $\varphi$ over the cell $\boldsymbol{V}$ :

$$
\langle\varphi\rangle=\frac{1}{|\boldsymbol{V}|} \int_{\boldsymbol{V}} \varphi(\boldsymbol{y}) d y, \quad\langle\varphi\rangle(\boldsymbol{x})=\frac{1}{|\boldsymbol{V}|} \int_{\boldsymbol{V}} \varphi(\boldsymbol{x}, \boldsymbol{y}) d y,
$$


and by $\langle\varphi\rangle_{f}$ (respectively, $\langle\varphi\rangle_{m}$ ) the mean value over the whole cell $\boldsymbol{V}$ of the field $\varphi$ only defined in or restricted to $\boldsymbol{V}_{f}$ (respectively, $\boldsymbol{V}_{m}$ ); i.e.,

$$
\langle\varphi\rangle_{f, m}=\frac{1}{|\boldsymbol{V}|} \int_{\boldsymbol{V}_{f, m}} \varphi(\boldsymbol{y}) d y, \quad\langle\varphi\rangle_{f, m}(\boldsymbol{x})=\frac{1}{|\boldsymbol{V}|} \int_{\boldsymbol{V}_{f, m}} \varphi(\boldsymbol{x}, \boldsymbol{y}) d y .
$$

Recalling that the center of the fiber is taken as the origin of the $\boldsymbol{y}$-coordinates, one has $\int_{\boldsymbol{V}_{f}} \boldsymbol{y} d y=\mathbf{0}$. Accordingly, after easy calculations, (51) can read as

$$
\begin{aligned}
& \int_{\Omega_{c}}\left\langle\boldsymbol{\sigma}^{0}\right\rangle \cdot \boldsymbol{\varepsilon}\left(\boldsymbol{u}^{*}\right) d x+\int_{\Omega_{d}}\left(\left\langle\boldsymbol{\sigma}^{0}\right\rangle \cdot \boldsymbol{\varepsilon}\left(\boldsymbol{u}^{*}\right)+\left\langle\boldsymbol{\sigma}^{0}\right\rangle_{f} \boldsymbol{e}_{3} \cdot \nabla \delta^{*}+\left\langle y_{\alpha} \boldsymbol{\sigma}^{0}\right\rangle_{f} \cdot \boldsymbol{\varepsilon}\left(\omega^{*} \boldsymbol{e}_{3} \wedge \boldsymbol{e}_{\alpha}\right)\right) d x \\
& =\int_{\Omega_{c}}\langle\rho\rangle \boldsymbol{g} \cdot \boldsymbol{u}^{*} d x+\int_{\Omega_{d}}\left(\langle\rho\rangle \boldsymbol{g} \cdot \boldsymbol{u}^{*}+\rho_{f} V_{f} g_{3} \delta^{*}\right) d x+\int_{\Gamma_{s}}\left(\boldsymbol{F} \cdot \boldsymbol{u}^{*}+V_{f} F_{3} \delta^{*}\right) d \Gamma,
\end{aligned}
$$

where $V_{f}$ denotes the volume fraction of the fibers; i.e.,

$$
V_{f}=\frac{\left|\boldsymbol{V}_{f}\right|}{|\boldsymbol{V}|}, \quad V_{m}=1-V_{f} .
$$

Remark 3. Let us note that $\omega^{*}$ does not appear in the right-hand side of (54). This is due to the assumption made on the applied forces, specifically that both the specific bulk forces $\boldsymbol{g}$ and the surface forces $\boldsymbol{F}$ do not depend on $\boldsymbol{y}$, and on the choice of the center of the fiber as the origin of the $\boldsymbol{y}$ coordinates.

Let us examine each term of the left-hand side of (54).

- For $\boldsymbol{x} \in \Omega_{c}$, by virtue of $(42),\left\langle\boldsymbol{\sigma}^{0}\right\rangle(\boldsymbol{x})$ reads as

$$
\left\langle\boldsymbol{\sigma}^{0}\right\rangle(\boldsymbol{x})=\mathrm{A}^{c} \boldsymbol{\varepsilon}(\boldsymbol{u})(\boldsymbol{x}),
$$

where $A^{c}$ denotes the (classical) homogenized stiffness tensor of the (perfectly bonded) composite; i.e.,

$$
\mathrm{A}_{i j k l}^{c}=\left\langle\mathrm{A}_{i j k l}+\mathrm{A}_{i j p q} \varepsilon\left(\chi^{k l}\right)_{p q}\right\rangle=\left\langle\mathrm{A}_{i j k l}-\mathrm{A} \boldsymbol{\varepsilon}\left(\chi^{i j}\right) \cdot \boldsymbol{\varepsilon}\left(\chi^{k l}\right)\right\rangle .
$$

The last equality above is obtained by using (32) with $\boldsymbol{w}=\chi^{k l}$. It allows us to check that $\mathrm{A}^{c}$ has the major symmetry $\mathrm{A}_{i j k l}^{c}=\mathrm{A}_{k l i j}^{c}$.

- For $\boldsymbol{x} \in \Omega_{d}$, by virtue of (49), $\left\langle\boldsymbol{\sigma}^{0}\right\rangle(\boldsymbol{x})$ reads as

$$
\left\langle\boldsymbol{\sigma}^{0}\right\rangle(\boldsymbol{x})=\mathrm{A}^{d} \boldsymbol{\varepsilon}(\boldsymbol{u})(\boldsymbol{x})+\left\langle\boldsymbol{S}^{3}\right\rangle \frac{\partial \delta}{\partial x_{3}}(\boldsymbol{x})+\left\langle\boldsymbol{T}^{3}\right\rangle \frac{\partial \omega}{\partial x_{3}}(\boldsymbol{x}),
$$

where $\mathrm{A}^{d}$ denotes the homogenized stiffness tensor of the debonded composite; i.e.,

$$
\mathrm{A}_{i j k l}^{d}=\left\langle\mathrm{A}_{i j k l}+\mathrm{A}_{i j p q} \varepsilon\left(\xi^{k l}\right)_{p q}\right\rangle=\left\langle\mathrm{A}_{i j k l}-\mathrm{A} \boldsymbol{\varepsilon}\left(\xi^{i j}\right) \cdot \boldsymbol{\varepsilon}\left(\boldsymbol{\xi}^{k l}\right)\right\rangle .
$$

The last equality above is obtained by using (34) with $\boldsymbol{w}=\boldsymbol{\xi}^{k l}$ and implies that $\mathrm{A}_{i j k l}^{d}=\mathrm{A}_{k l i j}^{d}$ for all $i, j, k, l$. The tensor $\mathrm{A}^{d}$ will be compared to the tensor $\mathrm{A}^{c}$ in 
the next section. Then, using (46) and the fact that $\langle\boldsymbol{y}\rangle_{f}=\mathbf{0}$, one gets $\left\langle\boldsymbol{T}^{3}\right\rangle=\mathbf{0}$ and finally

$$
\left\langle\boldsymbol{\sigma}^{0}\right\rangle(\boldsymbol{x})=\mathrm{A}^{d} \boldsymbol{\varepsilon}(\boldsymbol{u})(\boldsymbol{x})+\left\langle\boldsymbol{S}^{3}\right\rangle \frac{\partial \delta}{\partial x_{3}}(\boldsymbol{x}) .
$$

- For $\boldsymbol{x} \in \Omega_{d}$, using (49), the component $i$ of $\left\langle\boldsymbol{\sigma}^{0}\right\rangle_{f} \boldsymbol{e}_{3}(\boldsymbol{x})$ reads as

$$
\left\langle\sigma_{3 i}^{0}\right\rangle_{f}(\boldsymbol{x})=\left\langle\mathrm{A}_{3 i k l}^{f}+\mathrm{A}_{3 i r s}^{f} \varepsilon\left(\boldsymbol{\xi}^{k l}\right)_{r s}\right\rangle_{f} \varepsilon(\boldsymbol{u})_{k l}(\boldsymbol{x})+\left\langle S_{3 i}^{3}\right\rangle_{f} \frac{\partial \delta}{\partial x_{3}}(\boldsymbol{x})+\left\langle T_{3 i}^{3}\right\rangle_{f} \frac{\partial \omega}{\partial x_{3}}(\boldsymbol{x}) .
$$

Let us first show that

$$
\left\langle\mathrm{A}_{3 i k l}^{f}+\mathrm{A}_{3 i r s}^{f} \varepsilon\left(\xi^{k l}\right)_{r s}\right\rangle_{f}=\left\langle S_{k l}^{3}\right\rangle \delta_{i 3} .
$$

Considering (35) with $\boldsymbol{w}=\boldsymbol{\xi}^{k l}$ gives

$$
\left\langle A \boldsymbol{\varepsilon}\left(\boldsymbol{D}^{i}\right) \cdot \boldsymbol{\varepsilon}\left(\boldsymbol{\xi}^{k l}\right)\right\rangle+\left\langle\mathrm{A}_{3 i r s}^{f} \varepsilon\left(\boldsymbol{\xi}^{k l}\right)_{r s}\right\rangle_{f}=0 .
$$

Considering (34) with $k l$ instead of $i j$ and setting $\boldsymbol{w}=\boldsymbol{D}^{i}$ give

$$
\left\langle A \boldsymbol{\varepsilon}\left(\boldsymbol{D}^{i}\right) \cdot \boldsymbol{\varepsilon}\left(\boldsymbol{\xi}^{k l}\right)\right\rangle+\left\langle\mathrm{A}_{k l r s} \varepsilon\left(\boldsymbol{D}^{i}\right)_{r s}\right\rangle=0 .
$$

Therefore $\left\langle\mathrm{A}_{3 i r s}^{f} \varepsilon\left(\boldsymbol{\xi}^{k l}\right)_{r s}\right\rangle_{f}=\left\langle\mathrm{A}_{k l r s} \varepsilon\left(\boldsymbol{D}^{i}\right)_{r s}\right\rangle$ and hence

$$
\left\langle\mathrm{A}_{3 i k l}^{f}+\mathrm{A}_{3 i r s}^{f} \varepsilon\left(\boldsymbol{\xi}^{k l}\right)_{r s}\right\rangle_{f}=\left\langle\mathrm{A}_{k l r s} \varepsilon\left(\boldsymbol{D}^{i}\right)_{r s}\right\rangle+\left\langle\mathrm{A}_{3 i k l}^{f}\right\rangle_{f}=\left\langle S_{k l}^{i}\right\rangle,
$$

where the last equality is a direct consequence of the definition (44) of $\boldsymbol{S}^{i}$. Since $\boldsymbol{S}^{\alpha}=\mathbf{0}$, one gets (60).

Recalling now that $S_{3 \alpha}^{3}=0$ and $\left\langle\boldsymbol{T}^{3}\right\rangle_{f}=\left\langle\boldsymbol{T}^{3}\right\rangle=\mathbf{0}$, one finally obtains

$$
\left\langle\boldsymbol{\sigma}^{0}\right\rangle_{f} \boldsymbol{e}_{3}(\boldsymbol{x})=\left\langle\boldsymbol{S}^{3}\right\rangle \cdot \boldsymbol{\varepsilon}(\boldsymbol{u})(\boldsymbol{x}) \boldsymbol{e}_{3}+\left\langle S_{33}^{3}\right\rangle_{f} \frac{\partial \delta}{\partial x_{3}}(\boldsymbol{x}) \boldsymbol{e}_{3} .
$$

- The last term in the left-hand side of (54) can also read as

$$
\left\langle y_{\alpha} \boldsymbol{\sigma}^{0}\right\rangle_{f}(\boldsymbol{x}) \cdot \boldsymbol{\varepsilon}\left(\omega^{*} \boldsymbol{e}_{3} \wedge \boldsymbol{e}_{\alpha}\right)(\boldsymbol{x})=\left\langle\left(\boldsymbol{e}_{3} \wedge \boldsymbol{y}\right) \cdot \boldsymbol{e}_{q} \sigma_{q i}^{0}\right\rangle_{f}(\boldsymbol{x}) \frac{\partial \omega^{*}}{\partial x_{i}}(\boldsymbol{x}) .
$$

Using (49), one gets

$$
\begin{aligned}
\left\langle\left(\boldsymbol{e}_{3} \wedge \boldsymbol{y}\right) \cdot \boldsymbol{e}_{q} \sigma_{q i}^{0}\right\rangle_{f}(\boldsymbol{x})=\left\langle\left(\boldsymbol{e}_{3} \wedge \boldsymbol{y}\right) \cdot \boldsymbol{e}_{q}\left(\mathrm{~A}_{q i k l}^{f}+\mathrm{A}_{q i r s}^{f} \varepsilon\left(\boldsymbol{\xi}^{k l}\right)_{r s}\right)\right\rangle_{f} \varepsilon(\boldsymbol{u})_{k l}(\boldsymbol{x}) & \\
& +\left\langle\left(\boldsymbol{e}_{3} \wedge \boldsymbol{y}\right) \cdot \boldsymbol{e}_{q} S_{q i}^{3}\right\rangle_{f} \frac{\partial \delta}{\partial x_{3}}(\boldsymbol{x})+\left\langle\left(\boldsymbol{e}_{3} \wedge \boldsymbol{y}\right) \cdot \boldsymbol{e}_{q} T_{q i}^{3}\right\rangle_{f} \frac{\partial \omega}{\partial x_{3}}(\boldsymbol{x}) .
\end{aligned}
$$

Let us calculate the three effective coefficients appearing in the right side.

We first show that $\left\langle\left(\boldsymbol{e}_{3} \wedge \boldsymbol{y}\right) \cdot \boldsymbol{e}_{q}\left(\mathrm{~A}_{q i k l}^{f}+\mathrm{A}_{\text {qirs }}^{f} \varepsilon\left(\boldsymbol{\xi}^{k l}\right)_{r s}\right)\right\rangle_{f}=0$. First,

$$
\left\langle\left(\boldsymbol{e}_{3} \wedge \boldsymbol{y}\right) \cdot \boldsymbol{e}_{q} \mathrm{~A}_{q i k l}^{f}\right\rangle_{f}=\left(\boldsymbol{e}_{3} \wedge\langle\boldsymbol{y}\rangle_{f}\right) \cdot \boldsymbol{e}_{q} \mathrm{~A}_{q i k l}^{f}=0 .
$$

Then, recalling that $\boldsymbol{W}^{3}=\mathbf{0}$ and using (36) with $i=3$ and $\boldsymbol{w}=\boldsymbol{\xi}^{k l}$ give

$$
\left\langle\left(\boldsymbol{e}_{3} \wedge \boldsymbol{y}\right) \cdot \boldsymbol{e}_{q} \mathrm{~A}_{\text {qirs }}^{f} \varepsilon\left(\boldsymbol{\xi}^{k l}\right)_{r s}\right\rangle_{f}=0
$$


and hence the desired result.

Next we show that $\left\langle\left(\boldsymbol{e}_{3} \wedge \boldsymbol{y}\right) \cdot \boldsymbol{e}_{q} S_{q i}^{3}\right\rangle_{f}=0$. By virtue of (44), one has

$$
\left\langle\left(\boldsymbol{e}_{3} \wedge \boldsymbol{y}\right) \cdot \boldsymbol{e}_{q} S_{q i}^{3}\right\rangle_{f}=\left\langle\left(\boldsymbol{e}_{3} \wedge \boldsymbol{y}\right) \cdot \boldsymbol{e}_{q}\left(\mathrm{~A}_{q i 33}^{f}+\mathrm{A}_{q i r s}^{f} \varepsilon\left(\boldsymbol{D}^{3}\right)_{r s}\right)\right\rangle_{f} .
$$

Therefore, one can follow the same procedure as for the first coefficient. First,

$$
\left\langle\left(\boldsymbol{e}_{3} \wedge \boldsymbol{y}\right) \cdot \boldsymbol{e}_{q} \mathrm{~A}_{q i 33}^{f}\right\rangle_{f}=0 .
$$

Then, using (36) with $i=3$ and $\boldsymbol{w}=\boldsymbol{D}^{3}$ give

$$
\left\langle\left(\boldsymbol{e}_{3} \wedge \boldsymbol{y}\right) \cdot \boldsymbol{e}_{q} \mathrm{~A}_{q i r s}^{f} \varepsilon\left(\boldsymbol{D}^{3}\right)_{r s}\right\rangle_{f}=0
$$

and hence the desired result.

For the third effective coefficient, a direct calculation using (46) gives $\left\langle\left(\boldsymbol{e}_{3} \wedge \boldsymbol{y}\right)\right.$. $\left.\boldsymbol{e}_{q} T_{q i}^{3}\right\rangle_{f}=(\pi / 2) \mu_{f} R^{4} \delta_{i 3}$.

Therefore, one finally obtains

$$
\left\langle\left(\boldsymbol{e}_{3} \wedge \boldsymbol{y}\right) \cdot \boldsymbol{e}_{q} \sigma_{q i}^{0}\right\rangle_{f}(\boldsymbol{x})=\frac{\pi R^{4} \mu_{f}}{2|\boldsymbol{V}|} \frac{\partial \omega}{\partial x_{3}}(\boldsymbol{x}) \delta_{i 3} .
$$

Inserting (55), (59), (61) and (62) into (54), the variational equation (54) finally reads as

$$
\begin{gathered}
\int_{\Omega_{c}} A^{c} \boldsymbol{\varepsilon}(\boldsymbol{u}) \cdot \boldsymbol{\varepsilon}\left(\boldsymbol{u}^{*}\right) d x+\int_{\Omega_{d}} \frac{\pi R^{4} \mu_{f}}{2|\boldsymbol{V}|} \frac{\partial \omega}{\partial x_{3}} \frac{\partial \omega^{*}}{\partial x_{3}} d x \\
+\int_{\Omega_{d}}\left(A^{d} \boldsymbol{\varepsilon}(\boldsymbol{u}) \cdot \boldsymbol{\varepsilon}\left(\boldsymbol{u}^{*}\right)+\left\langle\boldsymbol{S}^{3}\right\rangle \cdot\left(\boldsymbol{\varepsilon}(\boldsymbol{u}) \frac{\partial \delta^{*}}{\partial x_{3}}+\frac{\partial \delta}{\partial x_{3}} \boldsymbol{\varepsilon}\left(\boldsymbol{u}^{*}\right)\right)+\left\langle S_{33}^{3}\right\rangle_{f} \frac{\partial \delta}{\partial x_{3}} \frac{\partial \delta^{*}}{\partial x_{3}}\right) d x \\
=\int_{\Omega}\langle\rho\rangle \boldsymbol{g} \cdot \boldsymbol{u}^{*} d x+\int_{\Omega_{d}} \rho_{f} V_{f} g_{3} \delta^{*} d x+\int_{\Gamma_{s}}\left(\boldsymbol{F} \cdot \boldsymbol{u}^{*}+V_{f} F_{3} \delta^{*}\right) d \Gamma
\end{gathered}
$$

The equality (63) must hold for all $\left(\boldsymbol{u}^{*}, \delta^{*}, \omega^{*}\right)$ such that the associated displacement field $v^{*}$ given by (50) is admissible. These admissibility conditions will be specified in the next subsection.

Proposition 3. The macroscopic displacement fields $(\boldsymbol{u}, \delta, \omega)$ are a stationary point of the following potential energy $\mathscr{P}^{0}$ :

$$
\begin{aligned}
\mathscr{P}^{0}\left(\boldsymbol{u}^{*}, \delta^{*}, \omega^{*}\right) \\
=\int_{\Omega_{c}} \frac{1}{2} A^{c} \boldsymbol{\varepsilon}\left(\boldsymbol{u}^{*}\right) \cdot \boldsymbol{\varepsilon}\left(\boldsymbol{u}^{*}\right) d x+\int_{\Omega_{d}} \frac{T}{2} \frac{\partial \omega^{*}}{\partial x_{3}} \frac{\partial \omega^{*}}{\partial x_{3}} d x \\
\quad+\int_{\Omega_{d}}\left(\frac{1}{2} \mathrm{~A}^{d} \boldsymbol{\varepsilon}\left(\boldsymbol{u}^{*}\right) \cdot \boldsymbol{\varepsilon}\left(\boldsymbol{u}^{*}\right)+\boldsymbol{\Sigma} \cdot \boldsymbol{\varepsilon}\left(\boldsymbol{u}^{*}\right) \frac{\partial \delta^{*}}{\partial x_{3}}+\frac{K}{2} \frac{\partial \delta^{*}}{\partial x_{3}} \frac{\partial \delta^{*}}{\partial x_{3}}\right) d x \\
\quad-\int_{\Omega}\langle\rho\rangle \boldsymbol{g} \cdot \boldsymbol{u}^{*} d x-\int_{\Omega_{d}} \rho_{f} V_{f} g_{3} \delta^{*} d x-\int_{\Gamma_{s}}\left(\boldsymbol{F} \cdot \boldsymbol{u}^{*}+V_{f} F_{3} \delta^{*}\right) d \Gamma,
\end{aligned}
$$


where the effective stiffness tensors $\mathrm{A}^{c}$ and $\mathrm{A}^{d}$, the effective stress tensor $\boldsymbol{\Sigma}$ and the effective rigidity coefficients $K$ and $T$ are obtained by solving the different cell problems. Specifically, $\mathrm{A}^{c}$ is given by (56), $\mathrm{A}^{d}$ by (58), $\Sigma=\left\langle S^{3}\right\rangle$ and $K=\left\langle S_{33}^{3}\right\rangle_{f}$, where $S^{3}$ is given by (47)-(48) and $T=\pi R^{4} \mu_{f} /(2|\boldsymbol{V}|)$.

Proof. It suffices to remark that (63) is equivalent to

$$
\left.\frac{d}{d h} \mathscr{P}^{0}\left(\boldsymbol{u}+h \boldsymbol{u}^{*}, \delta+h \delta^{*}, \omega+h \omega^{*}\right)\right|_{h=0}=0 .
$$

Hence, $\mathscr{P}^{0}$ can be seen as the effective potential energy of the composite body.

\section{Discussion and examples}

\subsection{Properties of the effective coefficients.}

Proposition 4. The effective rigidity tensor $\mathrm{A}^{c}$ of the perfectly bonded composite satisfies the minimization problem

$$
\text { for } \boldsymbol{\varepsilon}^{*} \in \mathbb{M}_{s}^{3}, \quad \mathrm{~A}^{c} \boldsymbol{\varepsilon}^{*} \cdot \boldsymbol{\varepsilon}^{*}=\min _{\boldsymbol{w} \in \mathscr{H}_{c}} \mathscr{E}^{c}(\boldsymbol{w}),
$$

where

$$
\mathscr{E}^{c}(\boldsymbol{w})=\left\langle\mathrm{A}\left(\boldsymbol{\varepsilon}^{*}+\boldsymbol{\varepsilon}(\boldsymbol{w})\right) \cdot\left(\boldsymbol{\varepsilon}^{*}+\boldsymbol{\varepsilon}(\boldsymbol{w})\right)\right\rangle .
$$

The effective rigidity tensor $\mathrm{A}^{d}$, the effective tensor $\boldsymbol{\Sigma}$ and the effective rigidity coefficient $K$ of the debonded composite satisfy the minimization problem

$$
\text { for } \boldsymbol{\varepsilon}^{*} \in \mathbb{M}_{s}^{3} \text { and } d^{*} \in \mathbb{R}, \quad \mathrm{A}^{d} \boldsymbol{\varepsilon}^{*} \cdot \boldsymbol{\varepsilon}^{*}+2 d^{*} \boldsymbol{\Sigma} \cdot \boldsymbol{\varepsilon}^{*}+K d^{* 2}=\min _{\boldsymbol{w} \in \mathscr{H}_{d}} \mathscr{E}^{d}(\boldsymbol{w}),
$$

where

$$
\begin{aligned}
\mathscr{E}^{d}(\boldsymbol{w})=\left\langle\mathrm{A}^{m}\left(\boldsymbol{\varepsilon}^{*}+\boldsymbol{\varepsilon}(\boldsymbol{w})\right) \cdot\right. & \left.\left(\boldsymbol{\varepsilon}^{*}+\boldsymbol{\varepsilon}(\boldsymbol{w})\right)\right\rangle_{m} \\
& +\left\langle\mathrm{A}^{f}\left(\boldsymbol{\varepsilon}^{*}+d^{*} \boldsymbol{e}_{3} \otimes \boldsymbol{e}_{3}+\boldsymbol{\varepsilon}(\boldsymbol{w})\right) \cdot\left(\boldsymbol{\varepsilon}^{*}+d^{*} \boldsymbol{e}_{3} \otimes \boldsymbol{e}_{3}+\boldsymbol{\varepsilon}(\boldsymbol{w})\right)\right\rangle_{f} .
\end{aligned}
$$

Therefore, there exist two positive constants $\alpha_{c}>0$ and $\alpha_{d}>0$ such that, for all $\boldsymbol{\varepsilon}^{*} \in \mathbb{M}_{s}^{3}$ and all $d^{*} \in \mathbb{R}$,

$$
\mathrm{A}^{c} \boldsymbol{\varepsilon}^{*} \cdot \boldsymbol{\varepsilon}^{*} \geq \alpha_{c} \boldsymbol{\varepsilon}^{*} \cdot \boldsymbol{\varepsilon}^{*}, \quad \mathrm{~A}^{d} \boldsymbol{\varepsilon}^{*} \cdot \boldsymbol{\varepsilon}^{*}+2 d^{*} \boldsymbol{\Sigma} \cdot \boldsymbol{\varepsilon}^{*}+K d^{* 2} \geq \alpha_{d}\left(\boldsymbol{\varepsilon}^{*} \cdot \boldsymbol{\varepsilon}^{*}+d^{* 2}\right) .
$$

Moreover, $\mathrm{A}^{c}$ and $\mathrm{A}^{d}$ are well ordered in the sense that

$$
\mathrm{A}^{c} \boldsymbol{\varepsilon}^{*} \cdot \boldsymbol{\varepsilon}^{*} \geq \mathrm{A}^{d} \boldsymbol{\varepsilon}^{*} \cdot \boldsymbol{\varepsilon}^{*} \text { for all } \boldsymbol{\varepsilon}^{*} \in \mathbb{M}_{s}^{3} .
$$

Proof. Let us prove the property of minimization for the debonded composite, the proof being similar for the perfectly bonded composite. Let $w^{*}$ be a minimizer 
of $\mathscr{E}^{d}$ over $\mathscr{H}_{d} ; \boldsymbol{w}^{*}$ is unique up to an element of $\mathscr{R}_{d}$ and satisfies the variational equation

$$
\left\langle\mathrm{A}^{m}\left(\boldsymbol{\varepsilon}^{*}+\boldsymbol{\varepsilon}\left(\boldsymbol{w}^{*}\right)\right) \cdot \boldsymbol{\varepsilon}(\boldsymbol{w})\right\rangle_{m}+\left\langle\mathrm{A}^{f}\left(\boldsymbol{\varepsilon}^{*}+d^{*} \boldsymbol{e}_{3} \otimes \boldsymbol{e}_{3}+\boldsymbol{\varepsilon}\left(\boldsymbol{w}^{*}\right)\right) \cdot \boldsymbol{\varepsilon}(\boldsymbol{w})\right\rangle_{f}=0
$$

for all $w \in \mathscr{H}_{d}$.

By linearity and using (34)-(35), one deduces that $\boldsymbol{w}^{*}(\boldsymbol{y})=\varepsilon_{i j}^{*} \xi^{i j}(\boldsymbol{y})+d^{*} \boldsymbol{D}^{3}(\boldsymbol{y})$. Moreover, using (68) with $\boldsymbol{w}=\boldsymbol{w}^{*}$ yields

$$
\begin{aligned}
\mathscr{E}^{d}\left(\boldsymbol{w}^{*}\right)=\left\langle\mathrm{A}^{m} \boldsymbol{\varepsilon}^{*} \cdot \boldsymbol{\varepsilon}^{*}-\mathrm{A}^{m} \boldsymbol{\varepsilon}\left(\boldsymbol{w}^{*}\right) \cdot \boldsymbol{\varepsilon}\left(\boldsymbol{w}^{*}\right)\right\rangle_{m} \\
\quad+\left\langle\mathrm{A}^{f}\left(\boldsymbol{\varepsilon}^{*}+d^{*} \boldsymbol{e}_{3} \otimes \boldsymbol{e}_{3}\right) \cdot\left(\boldsymbol{\varepsilon}^{*}+d^{*} \boldsymbol{e}_{3} \otimes \boldsymbol{e}_{3}\right)-\mathrm{A}^{f} \boldsymbol{\varepsilon}\left(\boldsymbol{w}^{*}\right) \cdot \boldsymbol{\varepsilon}\left(\boldsymbol{w}^{*}\right)\right\rangle_{f} \\
=\left\langle\mathrm{A} \boldsymbol{\varepsilon}^{*} \cdot \boldsymbol{\varepsilon}^{*}-\mathrm{A} \boldsymbol{\varepsilon}\left(\boldsymbol{w}^{*}\right) \cdot \boldsymbol{\varepsilon}\left(\boldsymbol{w}^{*}\right)\right\rangle+2 V_{f} \mathrm{~A}_{33 i j}^{f} \varepsilon_{i j}^{*} d^{*}+V_{f} \mathrm{~A}_{3333}^{f} d^{* 2} \\
=\left\langle\mathrm{A}_{i j k l}-\mathrm{A} \boldsymbol{\varepsilon}\left(\boldsymbol{\xi}^{i j}\right) \cdot \boldsymbol{\varepsilon}\left(\boldsymbol{\xi}^{k l}\right)\right\rangle \varepsilon_{i j}^{*} \varepsilon_{k l}^{*}+2\left(V_{f} \mathrm{~A}_{33 i j}^{f}-\left\langle\mathrm{A} \boldsymbol{\varepsilon}\left(\boldsymbol{\xi}^{i j}\right) \cdot \boldsymbol{\varepsilon}\left(\boldsymbol{D}^{3}\right)\right\rangle\right) \varepsilon_{i j}^{*} d^{*} \\
\quad+\left(V_{f} \mathrm{~A}_{3333}^{f}-\left\langle\mathrm{A} \boldsymbol{\varepsilon}\left(\boldsymbol{D}^{3}\right) \cdot \boldsymbol{\varepsilon}\left(\boldsymbol{D}^{3}\right)\right\rangle\right) d^{* 2} .
\end{aligned}
$$

Using (34) with $\boldsymbol{w}=\boldsymbol{D}^{3}$, (35) with $\boldsymbol{D}^{i}=\boldsymbol{w}=\boldsymbol{D}^{3}$ and (58), one gets $\mathscr{E}^{d}\left(\boldsymbol{w}^{*}\right)=\mathrm{A}^{d} \boldsymbol{\varepsilon}^{*} \cdot \boldsymbol{\varepsilon}^{*}+2\left(V_{f} \mathrm{~A}_{33 i j}^{f}+\left\langle\mathrm{A}_{i j k l} \varepsilon\left(\boldsymbol{D}^{3}\right)_{k l}\right\rangle\right) \varepsilon_{i j}^{*} d^{*}+\left\langle\mathrm{A}_{3333}^{f}+\mathrm{A}_{33 k l}^{f} \varepsilon\left(\boldsymbol{D}^{3}\right)_{k l}\right\rangle_{f} d^{* 2}$.

Then it suffices to use (44) with $i=3$ to obtain that $V_{f} \mathrm{~A}_{33 i j}^{f}+\left\langle\mathrm{A}_{i j k l} \varepsilon\left(D^{3}\right)_{k l}\right\rangle=$ $\left\langle S_{i j}^{3}\right\rangle=\Sigma_{i j}$ and $\left\langle\mathrm{A}_{3333}^{f}+\mathrm{A}_{33 k l}^{f} \varepsilon\left(\boldsymbol{D}^{3}\right)_{k l}\right\rangle_{f}=\left\langle S_{33}^{3}\right\rangle_{f}=K$. This yields (66).

We now prove the positivity of $\mathscr{E} d\left(\boldsymbol{w}^{*}\right)$. First, $\mathscr{C}^{d}\left(\boldsymbol{w}^{*}\right) \geq 0$ by definition and by the positivity of $\mathrm{A}^{m}$ and $\mathrm{A}^{f}$. We show that equality holds if and only if $\boldsymbol{\varepsilon}^{*}=\mathbf{0}$ and $d^{*}=0$. By the expression of $\mathscr{E} d\left(\boldsymbol{w}^{*}\right)$, equality holds if and only if

$$
\boldsymbol{\varepsilon}\left(\boldsymbol{w}^{*}\right)(\boldsymbol{y})=\left\{\begin{array}{cl}
-\boldsymbol{\varepsilon}^{*} & \text { for all } \boldsymbol{y} \in \boldsymbol{V}_{m}, \\
-\boldsymbol{\varepsilon}^{*}-d^{*} \boldsymbol{e}_{3} \otimes \boldsymbol{e}_{3} & \text { for all } \boldsymbol{y} \in \boldsymbol{V}_{f} .
\end{array}\right.
$$

But since $\varepsilon\left(\boldsymbol{w}^{*}\right)_{33}=0$, one gets $\varepsilon_{33}^{*}=d^{*}=0$. Accordingly, $\boldsymbol{\varepsilon}\left(\boldsymbol{w}^{*}\right)(\boldsymbol{y})=-\boldsymbol{\varepsilon}^{*}$ for all $\boldsymbol{y} \in \boldsymbol{V} \backslash \boldsymbol{I}$. But, since $\boldsymbol{w}^{*}$ is $\boldsymbol{V}$-periodic, one finally gets $\boldsymbol{\varepsilon}^{*}=\mathbf{0}$. Therefore the quadratic form $\mathrm{A}^{d} \boldsymbol{\varepsilon}^{*} \cdot \boldsymbol{\varepsilon}^{*}+2 d^{*} \boldsymbol{\Sigma} \cdot \boldsymbol{\varepsilon}^{*}+K d^{* 2}$ is definite positive on $\mathbb{M}_{s}^{3} \times \mathbb{R}$.

To prove that $\mathrm{A}^{c}$ and $\mathrm{A}^{d}$ are well ordered, let us take $d^{*}=0$. Then, by virtue of the minimization properties, one gets

$$
\begin{aligned}
& \mathrm{A}^{c} \boldsymbol{\varepsilon}^{*} \cdot \boldsymbol{\varepsilon}^{*}=\min _{\boldsymbol{w} \in \mathscr{H}_{c}}\left\langle\mathrm{~A}\left(\boldsymbol{\varepsilon}^{*}+\boldsymbol{\varepsilon}(\boldsymbol{w})\right) \cdot\left(\boldsymbol{\varepsilon}^{*}+\boldsymbol{\varepsilon}(\boldsymbol{w})\right)\right\rangle, \\
& \mathrm{A}^{d} \boldsymbol{\varepsilon}^{*} \cdot \boldsymbol{\varepsilon}^{*}=\min _{\boldsymbol{w} \in \mathscr{H}_{d}}\left\langle\mathrm{~A}\left(\boldsymbol{\varepsilon}^{*}+\boldsymbol{\varepsilon}(\boldsymbol{w})\right) \cdot\left(\boldsymbol{\varepsilon}^{*}+\boldsymbol{\varepsilon}(\boldsymbol{w})\right)\right\rangle .
\end{aligned}
$$

Since $\mathscr{H}_{c} \subset \mathscr{H}_{d}$, one obtains the desired inequality $\mathrm{A}^{c} \boldsymbol{\varepsilon}^{*} \cdot \boldsymbol{\varepsilon}^{*} \geq \mathrm{A}^{d} \boldsymbol{\varepsilon}^{*} \cdot \boldsymbol{\varepsilon}^{*}$ for all $\boldsymbol{\varepsilon}^{*}$ in $\mathbb{M}_{s}^{3}$.

4.2. The relevant functional framework of the effective model. Let us discuss here what are the relevant functional spaces so that the effective problem coming from the asymptotic analysis is well posed. The natural framework is the set of 
all functions with finite energy $\mathscr{P}^{0}$. Specifically, $\boldsymbol{u}^{*}$ must belong to $H^{1}\left(\Omega, \mathbb{R}^{3}\right)$ while $\delta^{*}$ and $\omega^{*}$ must belong to $H_{L}^{1}\left(\Omega_{d}\right)$, where

$$
H_{L}^{1}\left(\Omega_{d}\right)=\left\{\varphi: \varphi=0 \text { in } \Omega_{c}, \varphi \in L^{2}\left(\Omega_{d}\right), \frac{\partial \varphi}{\partial x_{3}} \in L^{2}\left(\Omega_{d}\right)\right\} .
$$

Accordingly, one can define as usual the trace of $\boldsymbol{u}^{*}$ on the boundary of $\Omega$ (and more generally on any sufficiently smooth surface included in $\bar{\Omega}$ ). Therefore, the Dirichlet boundary condition $\boldsymbol{u}^{*}=\mathbf{0}$ on $\Gamma_{c}$ has a sense. But this is not the case for the elements of $H_{L}^{1}\left(\Omega_{d}\right)$. Indeed, since one only controls its first derivative with respect to $x_{3}$, one can define the trace of such an element $\varphi$ on surfaces of the type $x_{3}=$ constant but not necessarily on surfaces with arbitrary orientations. Accordingly, the definition of the boundary conditions on $\Gamma_{c}$ and the continuity conditions at the interface between $\Omega_{c}$ and $\Omega_{d}$ need more developed arguments which are outside the scope of the present paper. As far as the linear part of the potential energy is concerned, the work done by the external forces is finite provided that the density $\boldsymbol{g}$ and $\boldsymbol{F}$ are sufficiently smooth. For the work of the specific forces, it suffices that $\boldsymbol{g}$ be in $L^{2}\left(\Omega ; \mathbb{R}^{3}\right)$ in order that both integrals over $\Omega$ and $\Omega_{d}$ be finite. The question is more delicate for $\boldsymbol{F}$. It is sufficient that $\boldsymbol{F}$ be in $L^{2}\left(\Gamma_{s} ; \mathbb{R}^{3}\right)$ in order that $\int_{\Gamma_{s}} \boldsymbol{F} \cdot \boldsymbol{u}^{*} d \Gamma<+\infty$. But, the term $\int_{\Gamma_{s} \cap{ }_{2} \Omega_{d}} F_{3} \delta^{*} d \Gamma$ makes sense only on the part of the boundary where either $F_{3}=0$ or $\delta^{*}$ is defined. Accordingly, we will assume that the following hypothesis holds:

Hypothesis 1. The given density of forces is such that $g \in L^{2}\left(\Omega ; \mathbb{R}^{3}\right)$ and $\boldsymbol{F} \in$ $L^{2}\left(\Gamma_{s} ; \mathbb{R}^{3}\right)$. Moreover, on the part $\Gamma_{s} \cap \partial \Omega_{d}, F_{3}=0$.

Finally, introducing the set of all kinematically admissible displacement fields

$$
\mathscr{C}^{0}=\left\{\left(\boldsymbol{u}^{*}, \delta^{*}, \omega^{*}\right) \in H^{1}\left(\Omega ; \mathbb{R}^{3}\right) \times H_{L}^{1}\left(\Omega_{d}\right)^{2}: \boldsymbol{u}^{*}=0 \text { on } \Gamma_{c}\right\},
$$

the effective problem can be formulated as follows:

$$
\text { find }(\boldsymbol{u}, \delta, \omega) \in \mathscr{C}^{0} \text { which minimizes } \mathscr{P}^{0} \text { over } \mathscr{C}^{0} \text {. }
$$

We are now in the position to establish the final result.

Proposition 5. Let $\mathscr{R}^{0}$ be the subset of $\mathscr{C}^{0}$ made of all displacement fields with null elastic energy:

$$
\mathscr{R}^{0}=\left\{\left(\boldsymbol{u}^{*}, \delta^{*}, \omega^{*}\right) \in \mathscr{C}^{0}: \boldsymbol{\varepsilon}\left(\boldsymbol{u}^{*}\right)=\mathbf{0} \text { in } \Omega, \frac{\partial \delta^{*}}{\partial x_{3}}=\frac{\partial \omega^{*}}{\partial x_{3}}=0 \text { in } \Omega_{d}\right\} .
$$

Then, if $\mathscr{R}^{0}=\{(\mathbf{0}, 0,0)\}$ and if the given forces $\boldsymbol{g}$ and $\boldsymbol{F}$ satisfy Hypothesis 1 , problem (70) admits a unique solution. 
Proof. Uniqueness is guaranteed by virtue of the assumption on $\mathscr{R}^{0}$ and of the positivity of the elastic energy. The existence is due to the smoothness assumption on the loading and to the positivity property (67) which ensures the coercivity.

Remark 4. The relative rotation of the fiber $\omega^{*}$ is not coupled with the macroscopic displacement field $\boldsymbol{u}^{*}$ and the sliding of the fiber $\delta^{*}$ in the elastic energy. Since $\omega^{*}$ does not appear in the work of the given external forces, one immediately obtains that the solution is such that $\partial \omega / \partial x_{3}=0$ in $\Omega_{d}$ and hence there does not exist a fiber torsional energy. But this property will no longer hold true if one changes some assumptions on the composite behavior or on the loading.

The solution $(\boldsymbol{u}, \delta)$ of the effective problem satisfies the following set of local equilibrium equations in $\Omega_{d}$ :

$$
\left\{\begin{array}{r}
\operatorname{div}\left(\mathrm{A}^{d} \boldsymbol{\varepsilon}(\boldsymbol{u})+\frac{\partial \delta}{\partial x_{3}} \boldsymbol{\Sigma}\right)+\langle\rho\rangle \boldsymbol{g}=\mathbf{0}, \\
\frac{\partial}{\partial x_{3}}\left(K \frac{\partial \delta}{\partial x_{3}}+\boldsymbol{\Sigma} \cdot \boldsymbol{\varepsilon}(\boldsymbol{u})\right)+V_{f} \rho_{f} g_{3}=0 .
\end{array}\right.
$$

These equations must be understood in the sense of distributions when the loading is not sufficiently smooth. The first one is a vectorial equation while the second one is scalar. Both are second-order partial differential equations and they are coupled by the term which involves the effective internal stress tensor $\boldsymbol{\Sigma}$.

4.3. Case of a regular hexagonal cell. Let $L$ be a characteristic length of the body, $\ell=3^{-1 / 4} \sqrt{2} L, \boldsymbol{a}=\ell \boldsymbol{e}_{1}, \boldsymbol{b}=\ell\left(\boldsymbol{e}_{1}+\sqrt{3} \boldsymbol{e}_{2}\right) / 2$ and $\boldsymbol{V}_{f}$ be the disk of center 0 and radius $R<\ell / 2$. Thus $\boldsymbol{V}$ is a regular hexagon centered at $\mathbf{0}$ with area $L^{2}$; see Figure 2. Since the material is isotropic, we can use the results of [Léné 1984] to obtain that $\mathrm{A}^{c}$ and $\mathrm{A}^{d}$ are positive transversely isotropic fourth-order tensors with axis $\boldsymbol{e}_{3}$. Therefore, $\mathrm{A}^{c}$ and $\mathrm{A}^{d}$ are such that, for all $\boldsymbol{\varepsilon} \in \mathbb{M}_{s}^{3}$,

$$
\begin{aligned}
& \mathrm{A}^{c} \boldsymbol{\varepsilon} \cdot \boldsymbol{\varepsilon}=A_{L}^{c} \varepsilon_{33}^{2}+\lambda_{L}^{c} \varepsilon_{33} \varepsilon_{\alpha \alpha}+\lambda_{T}^{c} \varepsilon_{\alpha \alpha}^{2}+2 \mu_{T}^{c} \varepsilon_{\alpha \beta} \varepsilon_{\alpha \beta}+2 \mu_{L}^{c} \varepsilon_{3 \alpha} \varepsilon_{3 \alpha}, \\
& \mathrm{A}^{d} \boldsymbol{\varepsilon} \cdot \boldsymbol{\varepsilon}=A_{L}^{d} \varepsilon_{33}^{2}+\lambda_{L}^{d} \varepsilon_{33} \varepsilon_{\alpha \alpha}+\lambda_{T}^{d} \varepsilon_{\alpha \alpha}^{2}+2 \mu_{T}^{d} \varepsilon_{\alpha \beta} \varepsilon_{\alpha \beta}+2 \mu_{L}^{d} \varepsilon_{3 \alpha} \varepsilon_{3 \alpha},
\end{aligned}
$$
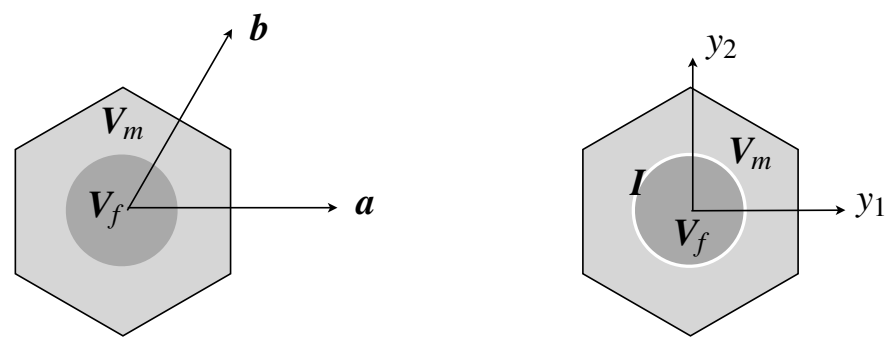

Figure 2. The case when the cell is a regular hexagon (left: bonded; right: debonded). 
where the ten moduli satisfy the following inequalities:

$$
\begin{gathered}
A_{L}^{c} \geq A_{L}^{d}>0, \quad \mu_{T}^{c} \geq \mu_{T}^{d}>0, \quad \mu_{L}^{c} \geq \mu_{L}^{d}>0, \\
A_{L}^{c}\left(\lambda_{T}^{c}+\mu_{T}^{c}\right)>\lambda_{L}^{c 2}, \quad A_{L}^{d}\left(\lambda_{T}^{d}+\mu_{T}^{d}\right)>\lambda_{L}^{d^{2}} .
\end{gathered}
$$

In the same manner, $\Sigma$ is transversely isotropic and hence can read

$$
\boldsymbol{\Sigma}=\sigma_{T}\left(\boldsymbol{e}_{1} \otimes \boldsymbol{e}_{1}+\boldsymbol{e}_{2} \otimes \boldsymbol{e}_{2}\right)+\sigma_{L} \boldsymbol{e}_{3} \otimes \boldsymbol{e}_{3}
$$

Let us compare the longitudinal shear moduli $\mu_{L}^{c}$ and $\mu_{L}^{d}$. They are given, respectively, by the two antiplane minimization cell problems

$$
\begin{aligned}
& \mu_{L}^{c}=\min _{\varphi \in H_{\#}^{1}(\boldsymbol{V})}\left\langle\mu\left(\nabla \varphi+\boldsymbol{e}_{1}\right) \cdot\left(\nabla \varphi+\boldsymbol{e}_{1}\right)\right\rangle, \\
& \mu_{L}^{d}=\min _{\varphi \in H_{\#}^{1}(\boldsymbol{V} \backslash \boldsymbol{I})}\left\langle\mu\left(\nabla \varphi+\boldsymbol{e}_{1}\right) \cdot\left(\nabla \varphi+\boldsymbol{e}_{1}\right)\right\rangle .
\end{aligned}
$$

The minimizers are the nonzero components $\chi_{3}^{13}$ and $\xi_{3}^{13}$ of $\chi^{13}$ and $\xi^{13}$. They satisfy

$$
\begin{array}{ll}
0=\left\langle\mu\left(\nabla \chi_{3}^{13}+\boldsymbol{e}_{1}\right) \cdot \nabla \varphi\right\rangle & \text { for all } \varphi \in H_{\#}^{1}(\boldsymbol{V}), \\
0=\left\langle\mu\left(\nabla \xi_{3}^{13}+\boldsymbol{e}_{1}\right) \cdot \nabla \varphi\right\rangle & \text { for all } \varphi \in H_{\#}^{1}(\boldsymbol{V} \backslash \boldsymbol{I}),
\end{array}
$$

where \# stands for periodic. It is easy to check that $\xi_{3}^{13}(\boldsymbol{y})=-y_{1}$ (plus an arbitrary constant) in $\boldsymbol{V}_{f}$. Therefore

$$
\mu_{L}^{d}=\left\langle\mu_{m}\left(\nabla \xi_{3}^{13}+\boldsymbol{e}_{1}\right) \cdot\left(\nabla \xi_{3}^{13}+\boldsymbol{e}_{1}\right)\right\rangle_{m}=\min _{\varphi \in H_{\#}^{1}\left(\boldsymbol{V}_{m}\right)}\left\langle\mu_{m}\left(\nabla \varphi+\boldsymbol{e}_{1}\right) \cdot\left(\nabla \varphi+\boldsymbol{e}_{1}\right)\right\rangle_{m} .
$$

In other words, the longitudinal shear modulus of the debonded composite is as if there were a hole instead of a fiber. Accordingly, $\mu_{L}^{c}$ and $\mu_{L}^{d}$ satisfy the following bounds:

$$
0<\mu_{L}^{d}<V_{m} \mu_{m}<\frac{1}{\frac{V_{m}}{\mu_{m}}+\frac{V_{f}}{\mu_{f}}}<\mu_{L}^{c}<V_{m} \mu_{m}+V_{f} \mu_{f},
$$

the last two inequalities corresponding to the classical Voigt and Reuss bounds.

In the particular case where the Poisson ratios of the fibers and the matrix equal 0 , then $\lambda_{f}=\lambda_{m}=0$. Moreover $\mu_{f}=E_{f}$ and $\mu_{m}=E_{m}, E_{f}$ and $E_{m}$ denoting the Young moduli of the fibers and the matrix. In this case, one easily deduces from (32), (34) and (35) that

$$
\chi^{33}=\xi^{33}=D^{3}=\mathbf{0}
$$

Therefore, one gets

$$
A_{L}^{c}=A_{L}^{d}=V_{m} E_{m}+V_{f} E_{f}, \quad \lambda_{L}^{c}=\lambda_{L}^{d}=0, \quad \sigma_{T}=0, \quad \sigma_{L}=K=V_{f} E_{f} .
$$

Let us remark that $\mathrm{A}^{c}$ and $\mathrm{A}^{d}$ are not strictly well ordered because $A_{L}^{c}=A_{L}^{d}$. 
4.4. Example. Let us finish this section by an example of application. We consider a cylinder $\Omega=S \times(0, L)$ whose cross-section $S$ is an open connected bounded subset of $\mathbb{R}^{2}$ and whose axis $\boldsymbol{e}_{3}$ corresponds to the vertical. This cylinder, submitted to the uniform gravity $\boldsymbol{g}=-g \boldsymbol{e}_{3}$, is fixed on its section $S \times\{L\}$ and free on all other boundaries $S \times\{0\}$ and $\partial S \times(0, L)$. It is made of a unidirectional composite, the fibers of which are periodically distributed according to a regular hexagonal lattice with axis $\boldsymbol{e}_{3}$. The Poisson ratios of the fibers and the matrix are equal to 0 . Accordingly, we are in the situation described at the end of the previous subsection; i.e.,

$$
\begin{aligned}
& \mathrm{A}^{c} \boldsymbol{\varepsilon} \cdot \boldsymbol{\varepsilon}=\langle E\rangle \varepsilon_{33}^{2}+\lambda_{T}^{c} \varepsilon_{\alpha \alpha}^{2}+2 \mu_{T}^{c} \varepsilon_{\alpha \beta} \varepsilon_{\alpha \beta}+2 \mu_{L}^{c} \varepsilon_{3 \alpha} \varepsilon_{3 \alpha}, \\
& \mathrm{A}^{d} \boldsymbol{\varepsilon} \cdot \boldsymbol{\varepsilon}=\langle E\rangle \varepsilon_{33}^{2}+\lambda_{T}^{d} \varepsilon_{\alpha \alpha}^{2}+2 \mu_{T}^{d} \varepsilon_{\alpha \beta} \varepsilon_{\alpha \beta}+2 \mu_{L}^{d} \varepsilon_{3 \alpha} \varepsilon_{3 \alpha}, \\
& \\
& \boldsymbol{\Sigma}=E_{f} V_{f} \boldsymbol{e}_{3} \otimes \boldsymbol{e}_{3}, \quad K=E_{f} V_{f} .
\end{aligned}
$$

Moreover, we assume that the fibers are debonded in the part $\Omega_{d}=S \times(0, \ell)$ and still bonded in the complementary part $\Omega_{c}=S \times(\ell, L)$ where $0<\ell<L$. Accordingly, the work of the gravity reads as

$$
f^{0}\left(\boldsymbol{u}^{*}, \delta^{*}\right)=-\int_{S \times(0, L)}\langle\rho\rangle g u_{3}^{*} d x-\int_{S \times(0, \ell)} \rho_{f} V_{f} g \delta^{*} d x,
$$

and the conditions of admissibility for the displacement fields are

$$
\begin{gathered}
\boldsymbol{u}^{*} \in H^{1}\left(S \times(0, L) ; \mathbb{R}^{3}\right), \quad\left(\delta^{*}, \omega^{*}\right) \in H_{L}^{1}(S \times(0, \ell))^{2}, \\
\boldsymbol{u}^{*}=\mathbf{0} \text { on } S \times\{L\}, \quad \delta^{*}=\omega^{*}=0 \text { on } S \times\{\ell\} .
\end{gathered}
$$

Therefore $\mathscr{R}^{0}=(\mathbf{0}, 0,0)$, we are in the situation of Proposition 5 and the effective problem admits a unique solution. Let us search for the solution under the form

$$
\boldsymbol{u}(\boldsymbol{x})=u\left(x_{3}\right) \boldsymbol{e}_{3}, \quad \delta(\boldsymbol{x})=\delta\left(\boldsymbol{x}_{3}\right), \quad \omega(\boldsymbol{x})=0 \quad \text { with } u(L)=0, \quad \delta(\ell)=0 .
$$

Then, the effective stress reads as

$$
\mathrm{A}^{c} \boldsymbol{\varepsilon}(\boldsymbol{u})(\boldsymbol{x})=\mathrm{A}^{d} \boldsymbol{\varepsilon}(\boldsymbol{u})(\boldsymbol{x})=\langle E\rangle u^{\prime}\left(x_{3}\right) \boldsymbol{e}_{3} \otimes \boldsymbol{e}_{3},
$$

where the prime denotes the derivative with respect to $x_{3}$. Inserting this form into (63), the variational effective problem becomes

$$
\begin{gathered}
0=\int_{S \times(0, \ell)}\left(\left(\langle E\rangle u^{\prime}+E_{f} V_{f} \delta^{\prime}\right) \frac{\partial u_{3}^{*}}{\partial x_{3}}+\langle\rho\rangle g u_{3}^{*}+E_{f} V_{f}\left(\delta^{\prime}+u^{\prime}\right) \frac{\partial \delta^{*}}{\partial x_{3}}+\rho_{f} V_{f} g \delta^{*}\right) d x \\
\quad+\int_{S \times(\ell, L)}\left(\langle E\rangle u^{\prime} \frac{\partial u_{3}^{*}}{\partial x_{3}}+\langle\rho\rangle g u_{3}^{*}\right) d x,
\end{gathered}
$$

and the equality must hold for all admissible $\left(\boldsymbol{u}^{*}, \delta^{*}\right)$. Taking first $\left(\boldsymbol{u}^{*}, \delta^{*}\right)$ of the same form as the expected solution, i.e., $\boldsymbol{u}^{*}(\boldsymbol{x})=v\left(x_{3}\right) \boldsymbol{e}_{3}$ and $\delta^{*}(\boldsymbol{x})=\varphi\left(x_{3}\right)$, we 
obtain the following one-dimensional variational problem for $(u, \delta)$ :

$$
\begin{aligned}
0=\int_{0}^{\ell}\left(\left(\langle E\rangle u^{\prime}+E_{f} V_{f} \delta^{\prime}\right) v^{\prime}+\langle\rho\rangle g v+E_{f} V_{f}\left(\delta^{\prime}+u^{\prime}\right) \varphi^{\prime}\right. & \left.+\rho_{f} V_{f} g \varphi\right) d x_{3} \\
& +\int_{\ell}^{L}\left(\langle E\rangle u^{\prime} v^{\prime}+\langle\rho\rangle g v\right) d x_{3},
\end{aligned}
$$

where the equality must hold for all $v \in H^{1}(0, L)$ such that $v(L)=0$ and all $\varphi \in$ $H^{1}(0, \ell)$ such that $\varphi(\ell)=0$. By standard arguments of calculus of variations, we find that $u$ and $\delta$ are the unique solution of the following boundary value problem:

$$
\begin{aligned}
& \text { in }(0, \ell): \quad\left\{\begin{array}{l}
\langle E\rangle u^{\prime \prime}+E_{f} V_{f} \delta^{\prime \prime}=\langle\rho\rangle g, \\
E_{f}\left(\delta^{\prime \prime}+u^{\prime \prime}\right)=\rho_{f} g ;
\end{array} \quad \text { in }(\ell, L): \quad\langle E\rangle u^{\prime \prime}=\langle\rho\rangle g ;\right. \\
& u^{\prime}(0)=\delta^{\prime}(0)=0 ; \quad \delta(\ell)=0, \quad \llbracket u \rrbracket(\ell)=0, \\
& \langle E\rangle \llbracket u^{\prime} \rrbracket(\ell)=E_{f} V_{f} \delta^{\prime}(\ell-) ; \quad u(L)=0 .
\end{aligned}
$$

After some calculations, we eventually find

$$
u^{\prime}\left(\boldsymbol{x}_{3}\right)=\left\{\begin{array}{ll}
\frac{\rho_{m}}{E_{m}} g x_{3}, & 0<x_{3}<\ell, \\
\frac{\langle\rho\rangle}{\langle E\rangle} g x_{3}, & \ell<x_{3}<L,
\end{array} \quad u(L)=0, \quad \delta\left(x_{3}\right)=\left(\frac{\rho_{f}}{E_{f}}-\frac{\rho_{m}}{E_{m}}\right) \frac{g}{2}\left(x_{3}^{2}-\ell^{2}\right) .\right.
$$

Conversely, the reader could verify that (77) is satisfied for any admissible $\left(\boldsymbol{u}^{*}, \delta^{*}\right)$ with $(u, \delta)$ given by $(80)$. Therefore, we have found the unique solution of the effective problem. Using (42) and (49), we can see the influence of the debonding on the repartition of the stresses inside the composite:

$$
\begin{aligned}
& \text { in } S \times(0, \ell): \quad \boldsymbol{\sigma}^{0}(\boldsymbol{x}, \boldsymbol{y})= \begin{cases}\frac{E_{f}}{\langle E\rangle}\langle\rho\rangle g x_{3} \boldsymbol{e}_{3} \otimes \boldsymbol{e}_{3} & \text { in } \boldsymbol{V}_{f}, \\
\frac{E_{m}}{\langle E\rangle}\langle\rho\rangle g x_{3} \boldsymbol{e}_{3} \otimes \boldsymbol{e}_{3} & \text { in } \boldsymbol{V}_{m},\end{cases} \\
& \text { in } S \times(\ell, L): \quad \boldsymbol{\sigma}^{0}(\boldsymbol{x}, \boldsymbol{y})= \begin{cases}\rho_{f} g x_{3} \boldsymbol{e}_{3} \otimes \boldsymbol{e}_{3} & \text { in } \boldsymbol{V}_{f}, \\
\rho_{m} g x_{3} \boldsymbol{e}_{3} \otimes \boldsymbol{e}_{3} & \text { in } \boldsymbol{V}_{m} .\end{cases}
\end{aligned}
$$

\section{Conclusion and perspectives}

We have shown that the effective behavior of a unidirectional composite material in the case where the fibers are debonded but still in contact with the matrix is formally similar to a generalized continuous medium whose kinematics contain not only the usual macroscopic displacement fields but also two scalar fields of internal variables describing the sliding and the rotation of the fibers. The two-scale procedure based on asymptotic expansions allowed us to formulate the effective problem giving the response of a composite body submitted to a mechanical loading. This problem can be formulated as the minimization of the effective potential energy of 
the composite body. This effective potential energy, difference of the effective elastic energy and the effective work of the applied forces, contains effective stiffness coefficients which are obtained by solving 12 elementary cell problems. Five of them can be solved in a closed form, the remaining seven requiring in general numerical computations. None of the problems are standard problems of the homogenization theory. Finally, the effective global problem leads to a system of coupled partial differential equations of second order which involve the kinematical fields.

The procedure was developed here in the particular case where the fibers and the matrix are linearly elastic isotropic materials with the assumption that the fibers remain in contact without friction with the matrix. We claim that it is possible to extend this work by removing some assumptions and enlarging the setting. For example, a first extension should be to consider prestresses in the composite and hence to develop the procedure in the case of an affine stress-strain relation. Another natural extension could be to consider more general and more realistic contact conditions between matrix and fibers: unilateral contact without friction or cohesive forces [Charlotte et al. 2006], for instance. The difficulty would be to solve nonlinear cell problems, and in such cases the effective behavior would no longer be described by a finite number of coefficients. An interesting mathematical challenge is to give a rigorous proof, by $\Gamma$-convergence for instance, that the effective behavior is really the one proposed here. It is a real issue because, as we have shown, the additional kinematical fields are less regular than the classical one. The consequences are that convergence could probably be proved only if the external forces satisfy certain smoothness conditions, and that the additional field should not satisfy arbitrary boundary conditions.

But the most interesting challenge is to introduce a law for the debonding evolution. Indeed, we have considered here that the domain where the fibers are debonded is given. But of course the real question is to find how this domain evolves with the loading. If we consider a Griffith-like assumption and suppose that debonding corresponds to an increase of the surface energy proportional to the new surface created [Bourdin et al. 2008], then the problem of debonding evolution will consist in finding when and how the potential energy is transformed into surface energy [Bilteryst and Marigo 2003]. If one adopts the global minimization principle proposed in [Francfort and Marigo 1993], then major mathematical difficulties will occur. Indeed, in the simplest case where the behavior of the material is described by two stiffness tensors, the damaged and the undamaged ones, it was shown in [Francfort and Marigo 1993] that the minimization energy problem does not admit classical solutions but must be relaxed to consider fine mixtures of damaged and undamaged material. In the present case the same phenomenon should probably also occur, but, because of the additional kinematical fields, its mathematical treatment should be much more difficult. 


\section{References}

[Abdelmoula and Marigo 2000] R. Abdelmoula and J.-J. Marigo, "The effective behavior of a fiber bridged crack", J. Mech. Phys. Solids 48:11 (2000), 2419-2444.

[Allaire 1992] G. Allaire, "Homogenization and two-scale convergence", SIAM J. Math. Anal. 23:6 (1992), 1482-1518.

[Bensoussan et al. 1978] A. Bensoussan, J.-L. Lions, and G. Papanicolaou, Asymptotic analysis for periodic structures, Studies in Mathematics and its Applications 5, North-Holland, Amsterdam, 1978.

[Berrehili and Marigo 2010] Y. Berrehili and J.-J. Marigo, "Modélisation en 2D du comportement d'un composite fibré à constituants décollés", Physical and Chemical News 53 (2010), 10-14.

[Bilteryst and Marigo 2003] F. Bilteryst and J.-J. Marigo, "An energy based analysis of the pull-out problem", Eur. J. Mech. A Solids 22:1 (2003), 55-69.

[Bouchelaghem et al. 2007] F. Bouchelaghem, A. Benhamida, and H. Dumontet, "Mechanical damage behaviour of an injected sand by periodic homogenization method", Computational Materials Science 38:3 (2007), 473-481.

[Bourdin et al. 2008] B. Bourdin, G. A. Francfort, and J.-J. Marigo, "The variational approach to fracture", J. Elasticity 91:1-3 (2008), 5-148.

[Caporale et al. 2006] A. Caporale, R. Luciano, and E. Sacco, "Micromechanical analysis of interfacial debonding in unidirectional fiber-reinforced composites", Computers \& Structures 84:31-32 (2006), 2200-2211.

[Charlotte et al. 2006] M. Charlotte, J. Laverne, and J.-J. Marigo, "Initiation of cracks with cohesive force models: A variational approach”, Eur. J. Mech. A Solids 25:4 (2006), 649-669.

[David et al. 2012] M. David, J.-J. Marigo, and C. Pideri, "Homogenized interface model describing inhomogeneities located on a surface", J. Elasticity 109:2 (2012), 153-187.

[Francfort and Marigo 1993] G. A. Francfort and J.-J. Marigo, "Stable damage evolution in a brittle continuous medium”, European J. Mech. A Solids 12:2 (1993), 149-189.

[Gonzàlez and LLorca 2007] C. Gonzàlez and J. LLorca, "Mechanical behavior of unidirectional fiber-reinforced polymers under transverse compression: Microscopic mechanisms and modeling", Composites Science and Technology 67:13 (2007), 2795-2806.

[Greco 2009] F. Greco, "Homogenized mechanical behavior of composite micro-structures including micro-cracking and contact evolution”, Engineering Fracture Mechanics 76:2 (2009), 182-208.

[Jendli et al. 2009] Z. Jendli, F. Meraghni, J. Fitoussi, and D. Baptiste, "Multi-scales modelling of dynamic behaviour for discontinuous fibre SMC composites", Composites Science and Technology 69:1 (2009), 97-103.

[Kulkarni et al. 2009] M. G. Kulkarni, P. H. Geubelle, and K. Matouš, "Multi-scale modeling of heterogeneous adhesives: Effect of particle decohesion”, Mechanics of Materials 41:5 (2009), 573583.

[Kushch et al. 2011] V. Kushch, S. Shmegera, and L. M. Jr., "Elastic interaction of partially debonded circular inclusions, II: Application to fibrous composite", Internat. J. Solids and Structures 48:1617 (2011), 2413-2421.

[Léné 1984] F. Léné, Contribution à l'étude des matériaux composites et de leur endommagement, thèse de doctorat d'état, Université Pierre et Marie Curie, Paris, 1984.

[Léné and Leguillon 1982] F. Léné and D. Leguillon, "Homogenized constitutive law for a partially cohesive composite material”, Internat. J. Solids and Structures 18:5 (1982), 443-458. 
[Marigo and Pideri 2011] J.-J. Marigo and C. Pideri, "The effective behavior of elastic bodies containing microcracks or microholes localized on a surface”, Int. J. Damage Mech. 20:8 (2011), 11511171.

[Marigo et al. 1987] J.-J. Marigo, P. Mialon, J.-C. Michel, and P. Suquet, "Plasticité et homogénéisation: Un exemple de prévision des charges limites d'une structure hétérogène périodique", J. Méc. Théor. Appl. 6 (1987), 47-75.

[Matouš and Geubelle 2006] K. Matouš and P. H. Geubelle, "Multiscale modelling of particle debonding in reinforced elastomers subjected to finite deformations", Internat. J. Numer. Methods Engrg. 65:2 (2006), 190-223.

[Michel et al. 1999] J. C. Michel, H. Moulinec, and P. Suquet, "Effective properties of composite materials with periodic microstructure: A computational approach", Comput. Methods Appl. Mech. Engrg. 172:1-4 (1999), 109-143.

[Moraleda et al. 2009] J. Moraleda, J. Segurado, and J. Llorca, "Effect of interface fracture on the tensile deformation of fiber-reinforced elastomers", Internat. J. Solids and Structures 46:25-26 (2009), 4287-4297.

[Sánchez-Palencia 1980] E. Sánchez-Palencia, Nonhomogeneous media and vibration theory, Lecture Notes in Physics 127, Springer, Berlin, 1980.

[Suquet 1982] P. Suquet, Plasticité et homogénéisation, thèse de doctorat d'état, Université Pierre et Marie Curie, Paris, 1982.

[Teng 2010] H. Teng, "Stiffness properties of particulate composites containing debonded particles", Internat. J. Solids and Structures 47:17 (2010), 2191-2200.

Received 31 Dec 2012. Revised 7 Apr 2013. Accepted 19 Jun 2013.

YAHYA BERREHILI: yberrehili@ensa.ump.ma

Equipe de Modélisation et Simulation Numérique, Université Mohamed 1er, Ecole Nationale des Sciences Appliquées, 60000 Oujda, Morocco

JEAN-JACQUES MARIGO: marigo@lms .polytechnique.fr

Laboratoire de Mécanique des Solides, École Polytechnique, CNRS, UMR 7649,

91128 Palaiseau cedex, France 


\section{EDITORIAL BOARD}

ANTONIO CARCATERRA ERIC A. CARLEN

FRANCESCO DELL'ISOLA

RAFFAELE ESPOSITO

ALBERT FANNJIANG

Gilles A. FrancFort

PiERANGElo MARCATI

JEAN-JACQUES MARIGO

PETER A. MARKOWICH MARTIN OSTOJA-STARZEWSKI

PIERRE SEPPECHER

DAVID J. STEIGMANN

PAUl STEINMANN

PierRe M. Suquet

\section{MANAGING EDITORS}

MICOL AMAR

CORRADO LATTANZIO

ANGELA MADEO

MARTIN OSTOJA-STARZEWSKI

\section{ADVISORY BOARD}

ADNAN AKAY

Holm AltenbaCH

MICOL AMAR

HARM ASKES

TEODOR ATANACKOVIĆ

VICTOR BERDICHEVSKY

GuY BouchitTÉ

ANDREA BRAIDES

ROBERTO CAMASSA

MAURO CARFORE

ERIC DARVE

FELIX DARVE

ANNA DE MASI

Gianpietro Del Piero

EMMANUELE Di BENEDETTO

BERNOLD FIEDLER

IRENE M. GAMBA

SERGEY GAVRILYUK

TIMOTHY J. HEALEY

DOMINIQUE JEULIN

ROGER E. KHAYAT

CORRADO LATTANZIO

ROBERT P. LIPTON

ANGELO LUONGO

ANGEla MadeO

JUAN J. MANFREDI

CARLO MARCHIORO

GÉrard A. MAUGin

ROBERTO NATALINI

PATRIZIO NEFF

ANDREY PIATNITSKI

ERrico Presutti

MARIO PUlVIRENTI

LuCiO RuSSO

Miguel A. F. SANJUAN

Patrick SElvadurai

ALEXANDER P. SEYRANIAN

MIROSLAV ŠILHAVÝ

GUIDO SWEERS

ANTOINETTE TORDESILLAS

LEV TRUSKINOVSKY

JUAN J. L. VELÁZQUEZ

VINCENZO VESPRI

ANGELO VULPIANI msp.org/memocs

Università di Roma “La Sapienza”, Italia

Rutgers University, USA

(CO-CHAIR) Università di Roma "La Sapienza", Italia

(TREASURER) Università dell'Aquila, Italia

University of California at Davis, USA

(CO-CHAIR) Université Paris-Nord, France

Università dell' Aquila, Italy

École Polytechnique, France

DAMTP Cambridge, UK, and University of Vienna, Austria

(CHAIR MANAGING EDITOR) Univ. of Illinois at Urbana-Champaign, USA

Université du Sud Toulon-Var, France

University of California at Berkeley, USA

Universität Erlangen-Nürnberg, Germany

LMA CNRS Marseille, France

Università di Roma “La Sapienza”, Italia

Università dell' Aquila, Italy

Université de Lyon-INSA (Institut National des Sciences Appliquées), France (CHAIR MANAGING EDITOR) Univ. of Illinois at Urbana-Champaign, USA

Carnegie Mellon University, USA, and Bilkent University, Turkey

Otto-von-Guericke-Universität Magdeburg, Germany

Università di Roma "La Sapienza”, Italia

University of Sheffield, UK

University of Novi Sad, Serbia

Wayne State University, USA

Université du Sud Toulon-Var, France

Università di Roma Tor Vergata, Italia

University of North Carolina at Chapel Hill, USA

Università di Pavia, Italia

Stanford University, USA

Institut Polytechnique de Grenoble, France

Università dell'Aquila, Italia

Università di Ferrara and International Research Center MEMOCS, Italia

Vanderbilt University, USA

Freie Universität Berlin, Germany

University of Texas at Austin, USA

Université Aix-Marseille, France

Cornell University, USA

École des Mines, France

University of Western Ontario, Canada

Università dell' Aquila, Italy

Louisiana State University, USA

Università dell' Aquila, Italia

Université de Lyon-INSA (Institut National des Sciences Appliquées), France

University of Pittsburgh, USA

Università di Roma "La Sapienza", Italia

Université Paris VI, France

Istituto per le Applicazioni del Calcolo "M. Picone", Italy

Universität Duisburg-Essen, Germany

Narvik University College, Norway, Russia

Università di Roma Tor Vergata, Italy

Università di Roma "La Sapienza", Italia

Università di Roma “Tor Vergata", Italia

Universidad Rey Juan Carlos, Madrid, Spain

McGill University, Canada

Moscow State Lomonosov University, Russia

Academy of Sciences of the Czech Republic

Universität zu Köln, Germany

University of Melbourne, Australia

École Polytechnique, France

Bonn University, Germany

Università di Firenze, Italia

Università di Roma La Sapienza, Italia

MEMOCS (ISSN 2325-3444 electronic, 2326-7186 printed) is a journal of the International Research Center for the Mathematics and Mechanics of Complex Systems at the Università dell'Aquila, Italy.

Cover image: "Tangle" by @ John Horigan; produced using the Context Free program (contextfreeart.org).

\section{PUBLISHED BY}

mathematical sciences publishers

nonprofit scientific publishing

http://msp.org/

(C) 2014 Mathematical Sciences Publishers 
Mathematics and Mechanics of Complex Systems vol. 2 no. 2

A mixed boundary value problem in potential theory for a bimaterial porous region: An application in the environmental geosciences

A. P. S. Selvadurai

Geometric degree of nonconservativity

Jean Lerbet, Marwa Aldowaji, Noël Challamel, Oleg N.

Kirillov, François Nicot and Félix Darve

Asymptotic analysis of small defects near a singular point in antiplane elasticity, with an application to the nucleation of a crack at a notch

Thi Bach Tuyet Dang, Laurence Halpern and Jean-Jacques Marigo

The homogenized behavior of unidirectional fiber-reinforced composite materials in the case of debonded fibers

Yahya Berrehili and Jean-Jacques Marigo

Statistically isotropic tensor random fields: Correlation structures

Anatoliy Malyarenko and Martin Ostoja-Starzewski

MEMOCS is a journal of the International Research Center for the Mathematics and Mechanics of Complex Systems at the Università dell' Aquila, Italy.

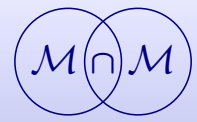

